\title{
Extensions of infinite partition regular systems
}

\author{
Neil Hindman* \\ Department of Mathematics \\ Howard University \\ Washington, DC 20059, U.S.A. \\ nhindman@aol.com
}

\author{
Imre Leader \\ Department of Pure Mathematics \\ and Mathematical Statistics \\ University of Cambridge \\ Cambridge, CB3 0WB, UK \\ leader@dpmms.cam.ac.uk
}

\author{
Dona Strauss \\ Department of Pure Mathematics \\ University of Leeds \\ Leeds LS2 9J2, UK \\ d.strauss@hull.ac.uk
}

Submitted: Jul 30, 2014; Accepted: May 8, 2015; Published: May 22, 2015

Mathematics Subject Classification: 05D10

\begin{abstract}
A finite or infinite matrix $A$ with rational entries (and only finitely many non-zero entries in each row) is called image partition regular if, whenever the natural numbers are finitely coloured, there is a vector $x$, with entries in the natural numbers, such that $A x$ is monochromatic. Many of the classical results of Ramsey theory are naturally stated in terms of image partition regularity.

Our aim in this paper is to investigate maximality questions for image partition regular matrices. When is it possible to add rows on to $A$ and remain image partition regular? When can one add rows but 'nothing new is produced'? What about adding rows and also new variables? We prove some results about extensions of the most interesting infinite systems, and make several conjectures.

Our most surprising positive result is a compatibility result for Milliken-Taylor systems, stating that (in many cases) one may adjoin one Milliken-Taylor system to a translate of another and remain image partition regular. This is in contrast to earlier results, which had suggested a strong inconsistency between different Milliken-Taylor systems. Our main tools for this are some algebraic properties of $\beta \mathbb{N}$, the Stone-Cech compactification of the natural numbers.
\end{abstract}

Keywords: Image partition regular; Ramsey Theory; central sets

*The first author acknowledges support received from the National Science Foundation (USA) via Grant DMS-1160566. 


\section{Introduction}

One of the earliest theorems in Ramsey Theory is Schur's Theorem [13], which says that if $\mathbb{N}$ is finitely coloured, then there exist $x_{0}$ and $x_{1}$ such that $\left\{x_{0}, x_{1}, x_{0}+x_{1}\right\}$ is monochromatic. Some time later, van der Waerden [15] proved that whenever $\mathbb{N}$ is finitely coloured and $k \in \mathbb{N}$, there is a monochromatic length $k$ arithmetic progression. Schur's Theorem and the length 4 version of van der Waerden's Theorem are precisely the assertions that the following two matrices are image partition regular.

$$
\left(\begin{array}{ll}
1 & 0 \\
0 & 1 \\
1 & 1
\end{array}\right) \quad\left(\begin{array}{ll}
1 & 0 \\
1 & 1 \\
1 & 2 \\
1 & 3
\end{array}\right)
$$

Here we say that a matrix $A$ with rational entries, and only finitely many non-zero entries in each row, is image partition regular or IPR if, whenever the natural numbers are finitely coloured, there is a vector $x$, with entries in the natural numbers, such that $A x$ is monochromatic (meaning that all the entries of $A x$ are natural numbers of the same colour).

In the finite case, the IPR matrices are well understood. Roughly speaking, they are the 'first-entries' matrices, meaning those for which all the rows whose first non-zero entry lies in a given column have the same entry in that column. See Section 2 for a precise statement about this.

[We have relegated to Section 2 background facts about finite matrices, and also about the Stone-Čech compactification $\beta \mathbb{N}$. The reader who is not especially interested in such things can just skip this section and refer back to it when necessary.]

In the infinite case, much less is known. As a 'trivial' example, note that, given a collection of finite matrices known to be IPR, it is possible to construct infinite IPR matrices. For example, if for $k \in \mathbb{N}$,

$$
A_{k}=\left(\begin{array}{cc}
1 & 0 \\
1 & 1 \\
\vdots & \vdots \\
1 & k
\end{array}\right) \text { and } B=\left(\begin{array}{cccc}
A_{2} & \mathrm{O} & \mathrm{O} & \ldots \\
\mathrm{O} & A_{3} & \mathrm{O} & \ldots \\
\mathrm{O} & \mathrm{O} & A_{4} & \ldots \\
\vdots & \vdots & \vdots & \ddots
\end{array}\right)
$$

then $B$ is IPR (since given any finite colouring there must be arbitrarily long arithmetic progressions in one of the colour classes, and thus arithmetic progressions of every length in that class).

What is probably the first nontrivial example of an infinite IPR matrix is the Finite Sums matrix. It was proved in [6] that whenever $\mathbb{N}$ is finitely coloured, there exists an infinite sequence $\left\langle x_{n}\right\rangle_{n=0}^{\infty}$ such that $F S\left(\left\langle x_{n}\right\rangle_{n=0}^{\infty}\right)$ is monochromatic, where

$$
F S\left(\left\langle x_{n}\right\rangle_{n=0}^{\infty}\right)=\left\{\sum_{n \in F} x_{n}: F \in \mathcal{P}_{f}(\omega)\right\}
$$

and $\mathcal{P}_{f}(\omega)$ is the set of finite nonempty subsets of $\omega$. We remark that this is the assertion that $\mathbf{F}$ is IPR, where all entries of $\mathbf{F}$ are 0 or 1 and for each $i<\omega, \sum_{j=0}^{\infty} f_{i, j} 2^{j}=i+1$. 
(We are following the custom of denoting the entry in row $i$ and column $j$ of a matrix by the lower case of the upper case letter which denotes the matrix.)

That is,

$$
\mathbf{F}=\left(\begin{array}{cccc}
1 & 0 & 0 & \ldots \\
0 & 1 & 0 & \ldots \\
1 & 1 & 0 & \ldots \\
0 & 0 & 1 & \ldots \\
1 & 0 & 1 & \ldots \\
0 & 1 & 1 & \ldots \\
1 & 1 & 1 & \ldots \\
\vdots & \vdots & \vdots & \ddots
\end{array}\right)
$$

However, most of the time we will not write matrices explicitly, being content to give the 'linear system' form (as in the ' $F S\left(\left\langle x_{n}\right\rangle_{n=0}^{\infty}\right)$ ' form above).

Using the Finite Sums Theorem as a tool, Milliken [11] and Taylor [14] independently established the fact that each of a whole class of matrices are IPR. We shall describe these matrices now.

Definition 1.1. Let $k \in \omega$ and let $\vec{a}=\left\langle a_{0}, a_{1}, \ldots, a_{k}\right\rangle$ be a sequence in $\mathbb{Z}$ such that $\vec{a} \neq \overrightarrow{0}$. The sequence $\vec{a}$ is compressed if and only if no $a_{i}=0$ and for each $i \in\{0,1, \ldots, k-1\}$, $a_{i} \neq a_{i+1}$. The sequence $c(\vec{a})=\left\langle c_{0}, c_{1}, \ldots, c_{m}\right\rangle$ is the compressed sequence obtained from $\vec{a}$ by first deleting all occurrences of 0 and then deleting any entry which is equal to its successor. Then $c(\vec{a})$ is called the compressed form of $\vec{a}$. And $\vec{a}$ is said to be a compressed sequence if $\vec{a}=c(\vec{a})$.

For example $c(\langle-2,0,-2,3,3,0,3,1,-2\rangle)=\langle-2,3,1,-2\rangle$. If $\vec{a}$ is an infinite sequence with finitely many nonzero entries, then $c(\vec{a})$ is defined analoguously, by first deleting the trailing 0's.

Definition 1.2. Let $k \in \omega$, let $\vec{a}=\left\langle a_{0}, a_{1}, \ldots, a_{k}\right\rangle$ be a compressed sequence in $\mathbb{Z} \backslash\{0\}$ with $a_{k}>0$, and let $\vec{x}=\left\langle x_{n}\right\rangle_{n=0}^{\infty}$. Then $M T(\vec{a}, \vec{x})=\left\{\sum_{i=0}^{k} a_{i} \sum_{t \in F_{i}} x_{t}: F_{0}, F_{1}, \ldots, F_{k} \in\right.$ $\mathcal{P}_{f}(\omega)$ and $\left.F_{0}<F_{1}<\ldots<F_{k}\right\}$, where for $F, G \in \mathcal{P}_{f}(\omega), F<G$ means $\max F<\min G$.

Note that the case $\vec{a}=\langle 1\rangle$ of the Milliken-Taylor theorem (Theorem 1.3 below) is precisely the Finite Sums Theorem.

Theorem 1.3. Let $k \in \omega$ and let $\vec{a}=\left\langle a_{0}, a_{1}, \ldots, a_{k}\right\rangle$ be a compressed sequence in $\mathbb{Z} \backslash\{0\}$ with $a_{k}>0$. Then whenever $\mathbb{N}$ is finitely coloured there exists an infinite sequence $\vec{x}=$ $\left\langle x_{n}\right\rangle_{n=0}^{\infty}$ such that $\operatorname{MT}(\vec{a}, \vec{x})$ is monochromatic.

Proof. If each $a_{i}>0$, this is [11, Theorem 2.2] and [14, Lemma 2.2]. The general case is a consequence of [8, Corollary 3.6].

In the sequel, we will occasionally need the matrix form of this. 
Definition 1.4. Let $k \in \omega$, let $\vec{a}=\left\langle a_{0}, a_{1}, \ldots, a_{k}\right\rangle$ be a compressed sequence in $\mathbb{Z} \backslash\{0\}$, and let $A$ be an $\omega \times \omega$ matrix. Then $A$ is an $M T(\vec{a})$-matrix if and only if the rows of $A$ are all rows $\vec{r} \in \mathbb{Z}^{\omega}$ such that $c(\vec{r})=\vec{a}$. The matrix $A$ is a Milliken-Taylor matrix if and only if it is an $M T(\vec{a})$-matrix for some $\vec{a}$.

Thus Theorem 1.3 asserts precisely that every Milliken-Taylor matrix is IPR. It will also be convenient to use the notation $\operatorname{Im}(\vec{x})$ for the set of the entries of a vector $\vec{x}$. So for example if $\vec{a}=\left\langle a_{0}, a_{1}, \ldots, a_{k}\right\rangle$ is a compressed sequence in $\mathbb{Z} \backslash\{0\}, A$ is an $M T(\vec{a})$-matrix, and $\vec{x} \in \mathbb{N}^{\omega}$, then $\operatorname{Im}(A \vec{x})$ is $M T(\vec{a}, \vec{x})$.

One of the major differences between finite and infinite IPR matrices is the following. It is a consequence of Theorem $2.2(\mathrm{~d})$, and the fact that given any finite colouring of $\mathbb{N}$, one colour class is central, that one colour class will contain an image of each finite IPR matrix. By way of contrast we have the following theorem of Deuber, Hindman, Leader and Lefmann.

Theorem 1.5. Let $k, m \in \omega$ let $\vec{a}=\left\langle a_{0}, a_{1}, \ldots, a_{k}\right\rangle$ and $\vec{b}=\left\langle b_{0}, b_{1}, \ldots, b_{m}\right\rangle$ be compressed sequences in $\mathbb{Z} \backslash\{0\}$ with $a_{k}>0$ and $b_{m}>0$, such that there is no positive rational $r$ with $\vec{a}=r \vec{b}$. Then there is a finite colouring of $\mathbb{N}$ such that there do not exist sequences $\vec{x}=\left\langle x_{n}\right\rangle_{n=0}^{\infty}$ and $\vec{y}=\left\langle y_{n}\right\rangle_{n=0}^{\infty}$ in $\mathbb{N}$ such that $M T(\vec{a}, \vec{x}) \cup M T(\vec{b}, \vec{y})$ is monochromatic.

Proof. [9, Theorem 3.1]. (The proof in the case all entries are positive was first done in [5, Theorems 3.2 and 3.3].)

In fact, if it is not the case that there is a positive rational $r$ such that $\vec{a}=r \vec{b}$, then there is a colouring as in Theorem 1.5 that has only two colours. (This can be seen in a fashion similar to the proof of [5, Theorem 3.14] where the same result is proved in the case that all entries are positive.)

The last of the special matrices with which we will be concerned is a DH-matrix. Roughly speaking, this is like the Finite Sums system, except that, instead of each $x_{n}$ being a fixed singleton, it can be taken from a given finite IPR system. The DH-matrix is IPR (see [4]), and so for example in any finite colouring of $\mathbb{N}$ one can find a sequence of arithmetic progressions $S_{1}, S_{2}, \ldots$, with $S_{i}$ having length $i$, such that all the finite sums obtained by adding up one member from each of finitely many of the $S_{i}$ have the same colour.

To be precise, we shall construct such a matrix as follows. First fix an enumeration $\left\langle B_{n}\right\rangle_{n=0}^{\infty}$ of the finite IPR matrices with rational entries. For each $n$, assume that $B_{n}$ is a $u(n) \times v(n)$ matrix. For each $i \in \mathbb{N}$, let $\overrightarrow{0}_{i}$ be the 0 vector with $i$ entries. Let $\mathbf{D}$ be an $\omega \times \omega$ matrix with all rows of the form $\vec{r}_{0} \frown \vec{r}_{1} \frown \vec{r}_{2} \frown \ldots$ where each $\vec{r}_{i}$ is either $\overrightarrow{0}_{v(i)}$ or is a row of $B_{i}$, and all but finitely many are $\overrightarrow{0}_{v(i)}$.

Definition 1.6. For each $n<\omega$ let $Y_{n} \in \mathcal{P}_{f}(\mathbb{Q})$. Then

$$
F S\left(\left\langle Y_{n}\right\rangle_{n=0}^{\infty}\right)=\left\{\sum_{n \in F} x_{n}: F \in \mathcal{P}_{f}(\omega) \text { and } x \in \mathrm{X}_{n \in F} Y_{n}\right\}
$$

Also, for $k \in \mathbb{N}, F S\left(\left\langle Y_{n}\right\rangle_{n=0}^{k}\right)=\left\{\sum_{n \in F} x_{n}: \emptyset \neq F \subseteq\{0,1, \ldots, k\}\right.$ and $\left.x \in \times_{n \in F} Y_{n}\right\}$. Given $F \in \mathcal{P}_{f}(\omega), \sum_{n \in F} Y_{n}=\left\{\sum_{n \in F} x_{n}: x \in X_{n \in F} Y_{n}\right\}$. 
Thus $F S\left(\left\langle Y_{n}\right\rangle_{n=0}^{\infty}\right)$ is all finite sums choosing at most one term from each $Y_{n}$. For each $n<\omega$, let $B_{n}$ be the $u(n) \times v(n)$ matrix used in the construction of $\mathbf{D}$. Define $k(0)=0$ and for $n \in \omega$, let $k(n+1)=k(n)+v(n)$. Assume that $\vec{x} \in \mathbb{Q}^{\omega}$. For each $n \in \omega$ let $\vec{y}_{n} \in \mathbb{Q}^{v(n)}$ be defined by $y_{n}(t)=x_{k(n)+t}$ and let $Y_{n}=\operatorname{Im}\left(B_{n} \vec{y}_{n}\right)$. Then $\operatorname{Im}(\mathbf{D} \vec{x})=F S\left(\left\langle Y_{n}\right\rangle_{n=0}^{\infty}\right)$.

The plan of the paper is as follows. In Section 3 we investigate the notion of maximal IPR matrices, meaning matrices such that no new row (not equal to any previous row) can be added in such a way that the resulting matrix is IPR. Finite matrices cannot have this property, and neither can F. We observe that matrices having all rows with a given constant row sum are maximal IPR and conjecture that these are the only examples of maximal IPR matrices.

We consider $\mathbf{F}$ in more detail in Section 4, giving a more restricted sense in which it is maximal (roughly speaking, this is the situation where we insist that the variables have disjoint support when written out in binary or similar).

In Section 5 we consider image maximality.

Definition 1.7. Let $t, u, v, w \in \mathbb{N} \cup\{\omega\}$, let $A$ be a $t \times u$ matrix and let $B$ be a $v \times w$ matrix. Then $A$ image dominates $B$ if and only if, for each $\vec{x} \in \mathbb{N}^{u}$ there exists $\vec{y} \in \mathbb{N}^{w}$ such that $\operatorname{Im}(B \vec{y}) \subseteq \operatorname{Im}(A \vec{x})$.

Notice that if $A$ image dominates $B$ and $A$ is IPR, then so is $B$. (The definition was designed so that this would be true.) Notice also that trivially, if $B$ is a finite IPR matrix, then the DH-matrix $\mathbf{D}$ image dominates $B$ (because $B=B_{n}$ for some $n$ ).

We say that a matrix $A$ is image maximal provided that whenever $B$ is an IPR matrix extending $A$, that is $B$ consists of $A$ with some rows added (but no new columns), then $A$ image dominates $B$.

We show that any IPR finite extension of $\mathbf{D}$ is in fact image dominated by $\mathbf{D}$ itself. We conjecture that $\mathbf{D}$ is image maximal, but have been unable to show this. This is perhaps the most tantalising of all the open questions.

Finally, in Section 6 we turn our attention to a more general notion. We say that an IPR matrix $A$ is universally image maximal provided that whenever $B$ is an IPR matrix that image dominates $A$, then $A$ image dominates $B$. In other words, this is like image maximality but we do not insist that $B$ is an extension of $A$.

This section contains what are our most surprising results. While we know that obviously $\mathbf{F}$ cannot be extended to an IPR matrix by adding on any Milliken-Taylor system except $\mathbf{F}$ itself, we show that one can add on translates of such matrices. In some sense this ought to be impossible, in light of Theorem 1.5. Similarly, it 'ought' to be the case that $\mathbf{D}$ is universally image maximal, but this turns out not to be the case: it will be shown in Theorem 6.6 that one can add a translate of what we might call a 'DHMT' system. The results of Section 6 do not depend on earlier material except some of the background presented in Section 2. So the reader who is interested primarily in these results can head directly to Section 6, referring to Section 2 as needed.

We do not know any examples of universally image maximal systems.

In this paper we shall always assume that any matrix that we consider has finitely many nonzero entries in each row. We also mention briefly that the matrices with which 
we will be dealing all have countably many rows and countably many columns, so of course the rows and columns could be rearranged so that they were all $u \times v$ matrices for some $u, v \in \mathbb{N} \cup\{\omega\}$. But it will be convenient, given $\omega \times \omega$ matrices $A$ and $B$ to discuss the matrices

$$
\left(\begin{array}{c}
A \\
B
\end{array}\right), \quad\left(\begin{array}{ll}
A & B
\end{array}\right), \quad \text { and } \quad\left(\begin{array}{cc}
A & \mathrm{O} \\
\mathrm{O} & B
\end{array}\right)
$$

where $\mathbf{O}$ is the $\omega \times \omega$ matrix with all zeroes. These are respectively $(\omega+\omega) \times \omega, \omega \times(\omega+\omega)$, and $(\omega+\omega) \times(\omega+\omega)$ matrices. However, we are of course always free to relabel these as $\omega \times \omega$ matrices, and we shall often implicitly do so.

\section{Background}

In his proof of a conjecture of Rado, Deuber [3] proved that certain matrices are IPR. (He called the set of entries in an image of such matrices an $(m, p, c)$-set. We shall have more to say about these later.) Deuber's matrices were special cases of first entries matrices. Since the concept of a first entries matrix has not turned out to be useful for infinite matrices, we shall restrict our definition to finite matrices.

Definition 2.1. Let $u, v \in \mathbb{N}$ and let $A$ be a $u \times v$ matrix with entries from $\mathbb{Q}$. Then $A$ is a first entries matrix if and only if no row of $A$ is $\overrightarrow{0}$ and whenever $i, j \in\{0,1, \ldots, u-1\}$ and

$$
\begin{aligned}
k & =\min \left\{t \in\{0,1, \ldots, v-1\}: a_{i, t} \neq 0\right\} \\
& =\min \left\{t \in\{0,1, \ldots, v-1\}: a_{j, t} \neq 0\right\},
\end{aligned}
$$

then $a_{i, k}=a_{j, k}>0$. An element $b$ of $\mathbb{Q}$ is a first entry of $A$ if and only if there is some row $i$ of $A$ such that $b=a_{i, k}$ where $k=\min \left\{t \in\{0,1, \ldots, v-1\}: a_{i, t} \neq 0\right\}$.

A few characterisations of finite IPR matrices were found in [7], including two computable characterisations. Several others have been found since. We list in the following theorem some characterisations that will be of interest to us in this paper. (We shall describe central sets later in this section.)

Theorem 2.2. Let $u, v \in \mathbb{N}$ and let $A$ be $a u \times v$ matrix with entries from $\mathbb{Q}$. The following statements are equivalent.

(a) $A$ is IPR.

(b) There exist $m \in \mathbb{N}$ and a $u \times m$ first entries matrix $B$ with entries from $\mathbb{Q}$ such that given any $\vec{y} \in \mathbb{N}^{m}$ there is some $\vec{x} \in \mathbb{N}^{v}$ with $A \vec{x}=B \vec{y}$.

(c) There exist $m \in \mathbb{N}$, a $u \times m$ first entries matrix $E$ with entries from $\omega$, and $c \in \mathbb{N}$ such that $c$ is the only first entry of $E$ and given any $\vec{y} \in \mathbb{N}^{m}$ there is some $\vec{x} \in \mathbb{N}^{v}$ with $A \vec{x}=E \vec{y}$.

(d) For every central set $C$ in $\mathbb{N}$, there exists $\vec{x} \in \mathbb{N}^{v}$ such that $A \vec{x} \in C^{u}$. 
(e) For each $\vec{r} \in \mathbb{Q}^{v} \backslash\{\overrightarrow{0}\}$ there exists $b \in \mathbb{Q} \backslash\{0\}$ such that $\left(\begin{array}{c}b \vec{r} \\ A\end{array}\right)$ is IPR.

(f) Whenever $C$ is a central subset of $\mathbb{N}$, and $m \in \mathbb{N},\left\{\vec{x} \in \mathbb{N}^{v}: A \vec{x} \in C^{u}\right.$, all entries of $\vec{x}$ are distinct and at least $m$ and entries of $A \vec{x}$ corresponding to distinct rows of $A$ are distinct $\}$ is central in $\mathbb{N}^{v}$.

Proof. These are respectively statements (a), (c), (f), (h), (j), and (m) of [10, Theorem 15.24] except that $(\mathrm{m})$ lacks the assertion that all entries of $\vec{x}$ are at least $m$. This follows because $\left\{\vec{x} \in \mathbb{N}^{v}\right.$ : all entries of $\vec{x}$ are at least $\left.m\right\}$ is an ideal of $\mathbb{N}^{v}$ and so it's intersection with any central set is central.

Note that as a consequence of Theorem 2.2(b), first entries matrices are IPR over $\mathbb{N}$.

As used in [3], given $m, p$, and $c$ in $\mathbb{N}$, Deuber's $(m, p, c)$-set is an image of a first entries matrix with $m$ columns, all first entries equal to $c$, all other entries from $\{-p,-p+$ $1, \ldots, p-1, p\}$, and all possible rows fitting this description. For example, a $(2,2,1)$-set is an image of the matrix

$$
\left(\begin{array}{cc}
1 & -2 \\
1 & -1 \\
1 & 0 \\
1 & 1 \\
1 & 2 \\
0 & 1
\end{array}\right)
$$

Most of the matrices with which we will deal will in fact have integer entries. However some of the results about finite matrices demand that non integer entries be allowed. For example, if $A=\left(\begin{array}{ll}1 & 1 \\ 1 & 2\end{array}\right)$ and $\vec{r}=\left(\begin{array}{ll}2 & 1\end{array}\right)$, then $A$ is IPR and the only $b$ such that $\left(\begin{array}{c}b \vec{r} \\ A\end{array}\right)$ is IPR is $b=\frac{1}{2}$, so that $b \vec{r}=\left(\begin{array}{ll}1 & \frac{1}{2}\end{array}\right)$. Thus we could not include statement (e) of Theorem 2.2 if we restricted to integer entries. (To verify that $b=\frac{1}{2}$, the reader can use [10, Theorem 15.24(b)].)

We conclude this section with a brief introduction to the algebraic structure of $\beta \mathbb{Z}$, both under addition and multiplication. (This structure will be used in some proofs in later sections.) For proofs of the assertions made here, see [10, Chapter 4].

If $(S, \cdot)$ is a discrete semigroup, we take the Stone-Cech compactification $\beta S$ of $S$ to be the set of ultrafilters on $S$, identifying the principle ultrafilters with the points of $S$ and thereby pretending that $S \subseteq \beta S$. (Similarly, for example, we identify an ultrafilter $p$ on $\mathbb{N}$ with the ultrafilter $\{A \subseteq \mathbb{Z}: A \cap \mathbb{N} \in p\}$ on $\mathbb{Z}$ and pretend that $\beta \mathbb{N} \subseteq \beta \mathbb{Z}$.) We write $S^{*}=\beta S \backslash S$. So $S^{*}$ is the set of nonprincipal ultrafilters on $S$.

Given a set $A \subseteq S, \bar{A}=\{p \in \beta S: A \in p\},\{\bar{A}: A \subseteq S\}$ is a basis for the topology on $\beta S$, and each $\bar{A}$ is clopen in $\beta S$. The operation on $S$ is extended to $\beta S$ so that for each $p \in \beta S$ the function $q \mapsto q \cdot p$ is continuous and for each $x \in S$ the function $q \mapsto x \cdot q$ is continuous. Given $p, q \in \beta S$ and $A \subseteq S, A \in p \cdot q$ if and only if $\left\{x \in S: x^{-1} A \in q\right\} \in p$, 
where $x^{-1} A=\{y \in S: x y \in A\}$. If the operation is denoted by + , one has that $A \in p+q$ if and only if $\{x \in S:-x+A \in q\} \in p$, where $-x+A=\{y \in S: x+y \in A\}$.

As with any compact Hausdorff right topological semigroup, $\beta S$ has idempotents and a smallest two-sided ideal $K(\beta S)$. Idempotents in the smallest ideal are called minimal. Given an idempotent $p \in \beta S, p$ is minimal if and only if $p \beta S p$ is a group. (We shall be using this in the context of $(\beta \mathbb{N},+)$ so that if $p$ is minimal, then $p+\beta \mathbb{N}+p$ is a group.)

The following lemma is [10, Lemma 4.14]. We include its simple proof to make this paper more self contained.

Lemma 2.3. Let $(S,+)$ be a semigroup, let $p$ be an idempotent in $\beta S$, let $A \in p$, and let $A^{\star}=\{x \in A:-x+A \in p\}$. Then $A^{\star} \in p$ and for any $x \in A^{\star},-x+A^{\star} \in p$.

Proof. Since $p$ is an idempotent, $A^{\star} \in p$. Let $x \in A^{\star}$, let $B=-x+A$, and let $C=$ $\{y \in B:-y+B \in p\}$. Then $B \in p$ and, since $p$ is an idempotent, $C \in p$. We claim that $C \subseteq-x+A^{\star}$. To see this, let $y \in C$. Then $y \in B$ so $x+y \in A$. Also $-(x+y)+A=-y+B \in p$ so $x+y \in A^{\star}$ and thus $y \in-x+A^{\star}$ as required.

Definition 2.4. Let $A \subseteq \mathbb{N}$. Then $A$ is central if and only if there is some minimal idempotent $p$ in $(\beta \mathbb{N},+)$ such that $A \in p$.

Definition 2.5. Let $u, v \in \mathbb{N} \cup\{\omega\}$ and let $A$ be a $u \times v$ matrix with entries from $\mathbb{Q}$.

(a) $A$ is centrally IPR if and only if whenever $C$ is a central set in $\mathbb{N}$, there exists $\vec{x} \in \mathbb{N}^{v}$ such that $A \vec{x} \in C^{u}$.

(b) $A$ is strongly centrally IPR if and only if whenever $C$ is a central set in $\mathbb{N}$, there exists $\vec{x} \in \mathbb{N}^{v}$ such that $A \vec{x} \in C^{u}$, the entries of $\vec{x}$ are distinct, and entries of $A \vec{x}$ corresponding to distinct rows of $A$ are distinct.

Notice that by Theorem 2.2(f), any finite IPR matrix is strongly centrally IPR.

Theorem 2.6. The matrices $\mathbf{D}$ and $\mathbf{F}$ are strongly centrally IPR.

Proof. We shall do the proof for $\mathbf{D}$. The proof for $\mathbf{F}$ is similar and simpler. In fact the result for $\mathbf{F}$ is a corollary of the result for $\mathbf{D}$ as can be seen by restricting to those $n<\omega$ for which $B_{n}$ consists of the first $v$ columns and first $2^{v}-1$ rows of $\mathbf{F}$ for some $v$.

The proof is a modification of [10, Theorem 16.16]. (This is essentially the result of the theorem of [4], which was restricted to $(m, p, c)$-sets.)

Let $\left\langle B_{n}\right\rangle_{n=0}^{\infty},\langle u(n)\rangle_{n=0}^{\infty}$, and $\langle v(n)\rangle_{n=0}^{\infty}$, be as in the construction of $\mathbf{D}$.

Let $C$ be central in $\mathbb{N}$ and pick a minimal idempotent $p$ in $(\beta \mathbb{N},+)$ such that $C \in p$. Let $C^{\star}=\{x \in C:-x+C \in p\}$ and note that by Lemma 2.3, if $x \in C^{\star}$, then $-x+C^{\star} \in p$. Pick by Theorem $2.2(\mathrm{f})$ some $\vec{x}(0) \in \mathbb{N}^{v(0)}$ such that all entries of $B_{0} \vec{x}(0)$ are in $C^{\star}$, the entries of $\vec{x}(0)$ are distinct, and entries of $B_{0} \vec{x}(0)$ corresponding to distinct rows of $B_{0}$ are distinct. Let $Y_{0}$ be the set of entries of $B_{0} \vec{x}(0)$.

Inductively, let $n \in \omega$ and assume that we have chosen $\vec{x}(k) \in \mathbb{N}^{v(k)}$ for each $k \in$ $\{0,1, \ldots, n\}$ so that, with $Y_{k}$ as the set of entries of $B_{k} \vec{x}(k)$, one has 
(1) $F S\left(\left\langle Y_{k}\right\rangle_{k=0}^{n}\right) \subseteq C^{\star}$;

(2) the entries of $\vec{x}(k)$ are distinct;

(3) entries of $B_{k} \vec{x}(k)$ corresponding to distinct rows of $B_{k}$ are distinct; and

(4) if $k<n$, then

$$
\begin{aligned}
& \max \left(\left\{x(k)_{i}: i \in\{0,1, \ldots, v(k)-1\}\right\} \cup Y(k)\right) \\
& <\min \left(\left\{x(k+1)_{i}: i \in\{0,1, \ldots, v(k+1)-1\}\right\} \cup Y(k+1)\right) .
\end{aligned}
$$

Let $m=\max \left(\left\{x(n)_{i}: i \in\{0,1, \ldots, v(n)-1\}\right\} \cup Y(n)\right)$ and let

$$
A=\{x \in \mathbb{N}: x>m\} \cap C^{\star} \cap \bigcap\left\{-a+C^{*}: a \in F S\left(\left\langle Y_{k}\right\rangle_{k=1}^{n}\right)\right\} .
$$

Then $A \in p$ so pick by Theorem 2.2(f) some $\vec{x} \in \mathbb{N}^{v(n+1)}$ such that all entries of $B_{n+1} \vec{x}(n+$ 1 ) are in $A$, the entries of $\vec{x}(n+1)$ are distinct and all at least $m+1$, and entries of $B_{n+1} \vec{x}(n+1)$ corresponding to distinct rows of $B_{n+1}$ are distinct. Let $Y_{n+1}$ be the set of entries of $B_{n+1} \vec{x}(n+1)$. Then $F S\left(\left\langle Y_{k}\right\rangle_{k=0}^{n+1}\right) \subseteq C^{\star}$.

\section{Extending the Finite Sums matrix}

We are concerned in this section with the general question, given an IPR matrix $A$, which matrices $B$ can be added so that $\left(\begin{array}{c}B \\ A\end{array}\right)$ is IPR. We saw in Theorem 2.2(e) that if $A$ is finite, it can be extended one row at a time practically at will.

By way of contrast, there exist finite kernel partition regular matrices which cannot be extended at all. (A $u \times v$ matrix $A$ is kernel partition regular if and only if whenever $\mathbb{N}$ is finitely coloured, there exists $\vec{x} \in \mathbb{N}^{v}$ whose entries are monochromatic such that $A \vec{x}=\overrightarrow{0}$.) Consider the matrix $A=\left(\begin{array}{ccc}1 & 1 & -1\end{array}\right)$. The assertion that $A$ is kernel partition regular is Schur's Theorem. The only way $A$ can be extended is by essentially repeating the same equation. That is, if $u, v$, and $w$ are rational numbers and $\left(\begin{array}{ccc}u & v & w \\ 1 & 1 & -1\end{array}\right)$ is kernel partition regular, then $u=v=-w$. (This can be seen by invoking Rado's Theorem [12, Satz IV].)

Definition 3.1. A matrix $A$ is maximal IPR provided it is IPR and if $\vec{r}$ is a row with finitely many nonzero entries which is not a row of $A$, then $\left(\begin{array}{c}\vec{r} \\ A\end{array}\right)$ is not IPR.

We give a trivial example of a maximal finite sums matrix in the following proposition.

Proposition 3.2. Let $c$ be a positive rational number, and let $A$ denote an $\omega \times \omega$ matrix over $\mathbb{Q}$ which contains all possible rows whose entries have a sum equal to $c$. Then $A$ is maximal IPR. 
Proof. We first observe that if $\vec{x} \in \mathbb{N}^{\omega}$ and $A \vec{x} \in \mathbb{N}^{\omega}$, then $\vec{x}$ has constant entries. To see this, let $m$ and $n$ be distinct elements of $\omega$, suppose that $x_{m}<x_{n}$, and pick $r \in \mathbb{N}$ such that $r>c x_{m}$. The vector in $\mathbb{Q}^{\omega}$ whose $m^{\prime}$ th entry is $c+r$ and whose $n^{\prime}$ th entry is $-r$, with all other entries being 0 , is a row of $A$. So $(c+r) x_{m}>r x_{n}$ and hence $c x_{m}>r\left(x_{n}-x_{m}\right) \geqslant r$, a contradiction.

Now suppose that the sum of the entries of $\vec{r}$ is $b \neq c$. We can define a finite colouring of $\mathbb{Q}^{+}$such that, for every $s \in \mathbb{Q}^{+}, b s$ and $c s$ have different colours. It follows that $\left(\begin{array}{c}\vec{r} \\ A\end{array}\right)$ cannot be IPR over $\mathbb{N}$. For example, observe that every element of $\mathbb{Q}^{+}$has a unique decomposition of the form $\prod_{i \in \mathbb{N}} p_{i}^{k_{i}}$ where $\left(p_{i}\right)_{i \in \mathbb{N}}$ denotes the sequence of prime numbers and each $k_{i} \in \mathbb{Z}$. We can choose a prime $p$ which occurs with different exponents $i$ and $j$ in the decomposition of $b$ and $c$ respectively. We can choose a prime $q>\max (|i|,|j|)$ and colour each $s \in \mathbb{Q}^{+}$by the value $(\bmod q)$ of the exponent of $p$ in the prime decomposition of $s$.

Conjecture 3.3. There are no maximal IPR matrices other than those given by Proposition 3.2 .

The reason for the title of the section is that the only results we have on the general question deal with extending the Finite Sums matrix. (Recall that we are denoting the Finite Sums matrix by F.) Thus, we are addressing the question of which matrices $B$ (of dimension $u \times \omega$ for some $u \in \mathbb{N} \cup\{\omega\})$ have the property that $\left(\begin{array}{c}B \\ \mathbf{F}\end{array}\right)$ is IPR. In the case that $u$ is finite, we can answer that question completely. (Recall that we are assuming that all the matrices which we consider have finitely many nonzero entries in each row, so that if $u$ is finite, then $B=\left(\begin{array}{ll}A & \mathrm{O}\end{array}\right)$ where $A$ is some finite matrix with $u$ rows and $\mathrm{O}$ is the $u \times \omega$ matrix with all zeroes.)

Theorem 3.4. Let $u, v \in \mathbb{N}$, let $A$ be $a u \times v$ matrix with rational entries, let $\mathbf{F}_{v}$ consist of the first $v$ columns and the first $2^{v}-1$ rows of $\mathbf{F}$, and let $\mathbf{O}$ be the $u \times \omega$ matrix with all zeroes. The following statements are equivalent.

(a) $\left(\begin{array}{c}A \\ \mathbf{F}\end{array}\right)$ is strongly centrally IPR.

(b) $\left(\begin{array}{c}A \quad \mathbf{O} \\ \mathbf{F}\end{array}\right)$ is centrally IPR.

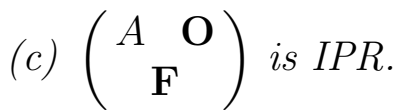

(d) $\left(\begin{array}{c}A \\ \mathbf{F}_{v}\end{array}\right)$ is IPR.

Proof. The only nontrivial implication is that (d) implies (a), so assume that $\left(\begin{array}{c}A \\ \mathbf{F}_{v}\end{array}\right)$ is IPR. Let $C$ be a central subset of $\mathbb{N}$ and pick a minimal idempotent $p \in \beta \mathbb{N}$ such that 
$C \in p$. Let $C^{\star}=\{x \in C:-x+C \in p\}$ and note that, by Lemma 2.3, if $x \in C^{\star}$, then $-x+C^{\star} \in p$. Then $C^{\star}$ is central, so by Theorem $2.2(\mathrm{f})$, pick $x_{0}, x_{1}, \ldots, x_{v-1}$, all distinct, such that

$$
\left(\begin{array}{c}
A \\
\mathbf{F}_{v}
\end{array}\right)\left(\begin{array}{c}
x_{0} \\
x_{1} \\
\vdots \\
x_{v-1}
\end{array}\right) \in\left(C^{\star}\right)^{u}
$$

and entries corresponding to distinct rows of $\left(\begin{array}{c}A \\ \mathbf{F}_{v}\end{array}\right)$ are distinct. Let $m$ be the maximum of all of these entries. Let

$$
\left.B=\{x \in \mathbb{N}: x>m\} \cap \bigcap-a+C^{\star}: a \text { is an entry of }\left(\begin{array}{c}
A \\
\mathbf{F}_{v}
\end{array}\right)\left(\begin{array}{c}
x_{0} \\
x_{1} \\
\vdots \\
x_{v-1}
\end{array}\right)\right\} .
$$

Then $B \in p$ so by [10, Theorem 5.14], pick a sequence $\left\langle H_{n}\right\rangle_{n=0}^{\infty}$ in $\mathcal{P}_{f}(\omega)$ such that for every $n \in \omega, \max H_{n}<\min H_{n+1}$ and, if $y_{n}=\sum_{t \in H_{n}} 2^{t}$, then $F S\left(\left\langle y_{n}\right\rangle_{n=0}^{\infty}\right) \subseteq B$. By discarding a few terms, we may assume that $\min H_{0} \geqslant m$. For $n \geqslant v$, let $x_{n}=y_{n}$. Then all entries of $\left(\begin{array}{cc}A & \mathbf{O} \\ \mathbf{F}\end{array}\right) \vec{x}$ are in $C$, entries of $\vec{x}$ are distinct, and entries of $\left(\begin{array}{cc}A & \mathbf{O} \\ \mathbf{F}\end{array}\right) \vec{x}$ corresponding to distinct rows of $\left(\begin{array}{cc}A & \mathbf{O} \\ \mathbf{F}\end{array}\right)$ are distinct.

The above proof in fact establishes something stronger than statement (a). For example, let $A$ be an $\omega \times \omega$ matrix with all rows beginning with 1 and then 2 and followed by 0's and 1's with finitely many 1's. The proof shows that $\left(\begin{array}{c}A \\ \mathbf{F}\end{array}\right)$ is strongly centrally IPR.

We do not know of any matrices that have entries not equal to either 0 or 1 arbitrarily far to the right and extend the Finite Sums matrix. We strongly suspect that the answer to the following question is "no", but cannot prove that it is.

Question 3.5. Let

$$
B=\left(\begin{array}{cccccc}
1 & 2 & 1 & 0 & 0 & \ldots \\
0 & 1 & 2 & 1 & 0 & \ldots \\
0 & 0 & 1 & 2 & 1 & \ldots \\
\vdots & \vdots & \vdots & \vdots & \vdots & \ddots
\end{array}\right)
$$

Is $\left(\begin{array}{c}B \\ \mathbf{F}\end{array}\right)$ IPR?

In the light of the following theorem, the matrix defined in Question 3.5 is the simplest possible matrix of this kind about which the question arises. In this theorem we let $\mathbf{F}^{\prime}$ be the submatrix of $\mathbf{F}$ consisting of the rows with at most two 1's. 
Theorem 3.6. Let $k \in \mathbb{N} \backslash\{1\}$ and let $a_{0}, a_{1}, a_{2}, \ldots, a_{k-1} \in \mathbb{Z}$, with $a_{0}$ and $a_{k-1}$ being non-zero. Let $A$ denote the $\omega \times \omega$ matrix whose $n$ 'th row has entries $a_{0}, a_{1}, \ldots, a_{k-1}$ in the columns indexed by $n, n+1, n+2, \ldots, n+k-1$ respectively, with all other entries being zero. Assume that $B=\left(\begin{array}{c}A \\ \mathbf{F}^{\prime}\end{array}\right)$ is IPR over $\mathbb{N}$. Then $a_{0}=a_{k-1}=1$.

Proof. Let $p$ be a prime number satisfying $p>\sum_{i=0}^{k-1}\left|a_{i}\right|$. Every $x \in \mathbb{N}$ can be expressed uniquely as $x=\sum_{n=0}^{\infty} e_{n}(x) p^{n}$, where each $e_{n}(x) \in\{0,1,2, \ldots, p-1\}$ and only finitely many are nonzero. We let $\operatorname{supp}(x)=\left\{n \in \omega: e_{n}(x) \neq 0\right\}$, let $m(x)=\min \operatorname{supp}(x)$, and let $M(x)=\max \operatorname{supp}(x)$. If $M(x)=0$, let $e_{-1}(x)=0$.

We define a finite colouring $\psi$ of $\mathbb{N}$, agreeing that $\psi(x)=\psi(y)$ if and only if

(1) $e_{m(x)}(x)=e_{m(y)}(y)$

(2) $e_{M(x)}(x)=e_{M(y)}(y)$,

(3) $e_{M(x)-1}(x)=e_{M(y)-1}(y)$, and

(4) $M(x) \equiv M(y)(\bmod 3)$,

Let $\vec{x} \in \mathbb{N}^{\omega}$ be a vector for which the entries of $B \vec{x}$ are monochromatic. Let $b, c$, and $d$ be the fixed values of $e_{m\left(x_{n}\right)}\left(x_{n}\right), e_{M\left(x_{n}\right)}\left(x_{n}\right)$, and $e_{M\left(x_{n}\right)-1}\left(x_{n}\right)$ respectively, for $n \in \omega$.

Let $m$ and $n$ be distinct elements of $\omega$. Then $m\left(x_{m}\right) \neq m\left(x_{n}\right)$ because $x_{m}, x_{n}$, and $x_{n}+x_{m}$ are all entries of $B \vec{x}$ and $2 b \not \equiv b(\bmod p)$. Now assume that $a_{0} \neq 1$. We claim that for each $l \in \omega, m\left(x_{l}\right)$ is not the minimum of $\left\{m\left(x_{l}\right), m\left(x_{l+1}\right), \ldots, m\left(x_{l+k-1}\right)\right\}$. Suppose instead $l \in \omega$ such that $m\left(x_{l}\right)=\min \left\{m\left(x_{l}\right), m\left(x_{l+1}\right), \ldots, m\left(x_{l+k-1}\right)\right\}$. Then $m\left(a_{0} x_{l}+a_{1} x_{l+1}+\ldots+a_{l+k-1} x_{k-1}\right)=m\left(x_{l}\right)$ because all digits at or to the right of $m\left(x_{l}\right)$ are zero in each $x_{l+i}$ such that $i \in\{1,2, \ldots, k-1\}$. But $a_{0} b \not \equiv b(\bmod p)$, so this is a contradiction. Now choose $r(0) \in\{1,2, \ldots, k-1\}$ such that $m\left(x_{r(0)}\right)<m\left(x_{0}\right)$. Inductively for $i \in \omega$, having chosen $r(i)$, choose $r(i+1) \in\{r(i)+1, r(i)+2, \ldots, r(i)+k-1\}$ such that $m\left(x_{r(i+1)}\right)<m\left(x_{r(i)}\right)$. Then $\left\langle m\left(x_{r(i)}\right)\right\rangle_{i=0}^{\infty}$ is a decreasing sequence in $\omega$, which is impossible. Consequently $a_{0}=1$.

We now claim that $M\left(x_{m}\right) \neq M\left(x_{n}\right)$. If $M\left(x_{m}\right)=M\left(x_{n}\right)$, then $M\left(x_{m}\right) \leqslant M\left(x_{m}+\right.$ $\left.x_{n}\right) \leqslant M\left(x_{m}\right)+1$. This implies that $M\left(x_{m}+x_{n}\right)=M\left(x_{m}\right)$ and hence that $c<\frac{p}{2}$. So the most significant digit in the base $p$ expansion of $x_{m}+x_{n}$ is $2 c$ or $2 c+1$, and this cannot be equal to $c$, a contradiction.

We observe that, if $x_{m}<x_{n}$ and $M\left(x_{n}\right)=s$, then $M\left(x_{m}\right) \leqslant s-3$. So $x_{n} \geqslant p^{s}$ and $x_{m}<p^{s-2}$, and hence $\frac{x_{n}}{x_{m}}>p^{2}$.

Assume that $a_{k-1} \neq 1$. Pick the first $n \geqslant k-1$ such that

$$
M\left(x_{n}\right)>\max \left\{M\left(x_{0}\right), M\left(x_{1}\right), \ldots, M\left(x_{k-2}\right)\right\} .
$$

Then $x_{n}=\max \left\{x_{n-k+1}, x_{n-k+2}, \ldots, x_{n}\right\}$. Let $t=\sum_{i=0}^{k-2} a_{i} x_{n-k+1+i}$. Then

$$
|t|<\left(\sum_{i=0}^{k-2}\left|a_{i}\right|\right) \frac{x_{n}}{p^{2}}<x_{n} \leqslant\left|a_{k-1}\right| x_{n} .
$$


Since $a_{k-1} x_{n}+t>0$, we must have $a_{k-1}>0$ and hence $a_{k-1} \geqslant 2$. Let $r=M\left(x_{n}\right)$. We have observed that, if $x_{i}<x_{n}$, then $x_{i}<p^{r-2}$. So $|t|<p^{r-1}$. We have that $M\left(a_{k-1} x_{n}+t\right)=r$, because $p^{r-1}<p^{r}-p^{r-1}<a_{k-1} x_{n}+t<p^{r+2}+p^{r-1}<p^{r+3}$. Therefore $a_{k-1} x_{n}+t=c p^{r}+d p^{r-1}+u$, where $0 \leqslant u<p^{r-1}$. We also have $x_{n}=c p^{r}+d p^{r-1}+v$, where $0 \leqslant v<p^{r-1}$. So $p^{r} \leqslant x_{n} \leqslant a_{k-1} x_{n}-x_{n}=u-v-t<2 p^{r-1}$, a contradiction.

\section{A maximal property of the Finite Sums matrix}

In this section we show that that the Finite Sums matrix, F, is maximal with respect to a particular notion of image partition regularity.

Definition 4.1. Let $u, v \in \mathbb{N} \cup\{\omega\}$ and let $A$ be a $u \times v$ matrix with entries from $\mathbb{Q}$. $A$ is rapidly IPR if and only if whenever $\mathbb{N}$ is finitely coloured and $p$ is a prime, there exists $\vec{x} \in \mathbb{N}^{v}$ such that the entries of $A \vec{x}$ are monochromatic and whenever $i+1<v$ and $s \in \omega$, if $p^{s} \leqslant x_{i}$, then $p^{s+8}$ divides $x_{i+1}$.

The intent of the definition is to force a relatively large gap between the highest order of one term of a relevant sum and the lowest order of another term when written in base $-p$.

We observe that $\mathbf{F}$, indeed all Milliken-Taylor matrices with final coefficient positive, are rapidly IPR. To see this, suppose that $M$ is a Milliken-Taylor matrix determined by the compressed sequence $\vec{a}=\left\langle a_{1}, a_{2}, \ldots, a_{k}\right\rangle$ in $\mathbb{Z}$ where $a_{k}>0$, let $p$ be a prime, and let $\mathbb{N}$ be finitely coloured. By Theorem 1.3 , pick a sequence $\left\langle x_{n}\right\rangle_{n=0}^{\infty}$ in $\mathbb{N}$ such that $\operatorname{MT}\left(\vec{a},\left\langle x_{n}\right\rangle_{n=0}^{\infty}\right)$ is monochromatic. Let $H_{0}=\{0\}$ and let $y_{0}=x_{0}$. Inductively, let $n \in \mathbb{N}$ and assume we have chosen $\left\langle H_{m}\right\rangle_{m=0}^{n-1}$ in $\mathcal{P}_{f}(\omega)$ and $\left\langle y_{m}\right\rangle_{m=0}^{n-1}$ in $\mathbb{N}$ such that

(1) for each $m \in\{0,1, \ldots, n-1\}, y_{m}=\sum_{t \in H_{m}} x_{t}$;

(2) for each $m \in\{1,2, \ldots, n-1\}$, $\min H_{m}>\max H_{m-1}$; and

(3) for each $m \in\{1,2, \ldots, n-1\}$ and each $s \in \omega$, if $p^{s} \leqslant y_{m-1}$, then $p^{s+8}$ divides $y_{m}$.

Pick the largest $s \in \omega$ such that $p^{s} \leqslant y_{n-1}$ and let $r=\max H_{n-1}$. Pick a set $H_{n} \subseteq\{m \in$ $\mathbb{N}: m>r\}$ such that $\left|H_{n}\right|=p^{s+8}$ and for any $i$ and $j$ in $H_{n}, x_{i} \equiv x_{j}\left(\bmod p^{s+8}\right)$. Let $y_{n}=\sum_{t \in H_{n}} x_{t}$. The sequence $\left\langle y_{n}\right\rangle_{n=0}^{\infty}$ having been chosen, it is routine to verify that $M T\left(\vec{a},\left\langle n_{n}\right\rangle_{n=0}^{\infty}\right) \subseteq M T\left(\vec{a},\left\langle x_{n}\right\rangle_{n=0}^{\infty}\right)$.

In particular, since a Milliken-Taylor matrix determined by the compressed sequence $\vec{a}=\left\langle a_{1}, a_{2}, \ldots, a_{k}\right\rangle$ with $k>1$ is not centrally IPR by Theorem 1.5 , we see that rapidly IPR matrices need not be centrally IPR. On the other hand, the matrix $A=\left(\begin{array}{ll}0 & 1 \\ 1 & 2\end{array}\right)$ is strongly centrally IPR, since it is a first entries matrix, but is not rapidly IPR. To see the latter assertion, colour $x \in \mathbb{N}$ by whether $\max \left\{t \in \omega: 2^{t} \leqslant x\right\}$ is even or odd and let $p=2$.

We shall show in Theorem 4.5 that $\mathbf{F}$ is maximal among rapidly IPR matrices with integer entries. To do this we will utilize the representation of integers to negative bases, 
as was done in [9]. (The main advantage of using negative bases is that one can eliminate borrowing when subtracting numbers by an appropriate choice of terms.)

We omit the routine proof of the following lemma.

Lemma 4.2. Let $p \in \mathbb{N} \backslash\{1\}$, let $s \in \omega$, and let $x \in \mathbb{Z} \backslash\{0\}$. There exist $\left\langle d_{i}\right\rangle_{i=0}^{s}$ with each $d_{i} \in\{0,1, \ldots, p-1\}$ and $d_{s}>0$ such that $x=\sum_{i=0}^{s} d_{i}(-p)^{i}$ if and only if

(1) $s$ is even and $\frac{p^{s}+p}{p+1} \leqslant x \leqslant \frac{p^{s+2}-1}{p+1}$ or

(2) $s$ is odd and $\frac{-p^{s+2}+p}{p+1} \leqslant x \leqslant \frac{-p^{s}-1}{p+1}$.

It follows immediately from Lemma 4.2 that given $p \in \mathbb{N} \backslash\{1\}$ and $x \in \mathbb{Z}$, there is a unique choice of $\left\langle d_{i}\right\rangle_{i=0}^{\infty}$ with each $d_{i} \in\{0,1, \ldots, p-1\}$ such that $x=\sum_{i=0}^{\infty} d_{i}(-p)^{i}$. In the following definition we suppress the dependence of $d_{i}(x)$ and $\operatorname{supp}(x)$ on $p$ because we will be using only one value of $p$ in the proof of Theorem 4.5.

Definition 4.3. Let $x \in \mathbb{Z}$ and let $p \in \mathbb{N} \backslash\{1\}$.

(a) $\left\langle d_{i}(x)\right\rangle_{i=0}^{\infty}$ is the unique sequence in $\{0,1, \ldots, p-1\}$ such that $x=\sum_{i=0}^{\infty} d_{i}(x)(-p)^{i}$.

(b) $\operatorname{supp}(x)=\left\{i \in \omega: d_{i}(x) \neq 0\right\}$.

Lemma 4.4. Let $x \in \mathbb{N}$, let $a \in \mathbb{Z} \backslash\{0\}$, and let $p \in \mathbb{N}$ with $p>|a|$. Let $s=\max \operatorname{supp}(x)$ and let $r=\max \operatorname{supp}(a x)$.

(1) $p^{s-2}<x<p^{s+1}$.

(2) If $a>0$, then $s \leqslant r \leqslant s+2$.

(3) If $a<0$, then $s-1 \leqslant r \leqslant s+1$.

Proof. We have by Lemma 4.2 that

$$
\frac{p^{s}+p}{p+1} \leqslant x \leqslant \frac{p^{s+2}-1}{p+1} .
$$

Conclusion (1) then follows immediately. Conclusions (2) and (3) are derived in the same way. We will do the computations for (3), since they are slightly more complicated.

So assume $a<0$. By $(*)$ we have $a \frac{p^{s+2}-1}{p+1} \leqslant a x \leqslant a \frac{p^{s}+p}{p+1}$ and by Lemma 4.2 we have that $\frac{-p^{r+2}+p}{p+1} \leqslant a x \leqslant \frac{-p^{r}-1}{p+1}$. Thus we have that $a\left(p^{s+2}-1\right) \leqslant-p^{r}-1$ and $-p^{r+2}+p \leqslant a\left(p^{s}+p\right)$. Consequently $p^{r}+1 \leqslant|a|\left(p^{s+2}-1\right)<p^{s+3}-p$ and $p^{s}+p \leqslant|a|\left(p^{s}+p\right) \leqslant p^{r+2}-p$. Since $p^{r}+p+1<p^{s+3}$, we have that $r<s+3$ so, since $r$ is odd (because $a x<0$ ), $r \leqslant s+1$. Since $p^{s}+2 p \leqslant p^{r+2}, s<r+2$ so $r \geqslant s-1$.

In the following theorem we will show that one cannot add any row $\vec{r}$ to $\mathbf{F}$ whose nonzero entries in order are $a_{1}, a_{2}, \ldots, a_{k}$ and remain rapidly IPR unless $a_{1}=a_{2}=\ldots=$ $a_{k}=1$ (in which case $\vec{r}$ is already a row of $\mathbf{F}$ ). By way of contrast, by Theorem 3.4, if any $a_{i}=1$, then $\left(\begin{array}{c}\vec{r} \\ \mathbf{F}\end{array}\right)$ is strongly centrally IPR (because the columns can be rearranged so that $\left(\begin{array}{c}\vec{r} \\ \mathbf{F}\end{array}\right)$ extends a finite first entries matrix). 
Theorem 4.5. The Finite Sums matrix $\mathbf{F}$ is maximal among rapidly IPR matrices with integer entries.

Proof. Suppose not and let $\vec{r} \in \mathbb{Z}^{\omega}$ with finitely many nonzero entries and not all entries in $\{0,1\}$ such that $B=\left(\begin{array}{c}\vec{r} \\ \mathbf{F}\end{array}\right)$ is rapidly IPR. Assume that the nonzero entries of $\vec{r}$ are $a_{1}, a_{2}, \ldots, a_{k}$ in order and that they occur in columns $j(1), j(2), \ldots, j(k)$ respectively. Let $r=\min \left\{i \in\{1,2, \ldots, k\}: a_{i} \neq 1\right\}$. Pick a prime $p$ such that $k<p$ and for all $i \in\{1,2, \ldots, k\}, 2\left|a_{i}\right|<p$.

For $x \in \mathbb{Z} \backslash\{0\}$, define $f(x)=d_{\min } \operatorname{supp}(x)(x)$, the least significant digit of $x$ in the base $-p$ expansion. For $x \in \mathbb{Z} \backslash\{0\}$ with $\max \operatorname{supp}(x)=s \geqslant 3$, define $\phi(x)=\left\langle u_{0}, u_{1}, u_{2}, u_{3}\right\rangle \in$ $\{1,2, \ldots, p-1\} \times\{0,1, \ldots, p-1\}^{3}$, where for $i \in\{0,1,2,3\}, u_{i}=d_{s-i}(x)$.

For $x \in \mathbb{Z} \backslash\{0\}$ and $\left\langle v, u_{0}, u_{1}, u_{2}, u_{3}\right\rangle \in\{1,2, \ldots, p-1\}^{2} \times\{0,1, \ldots, p-1\}^{3}$, let

$$
\begin{aligned}
G_{v, u_{0}, u_{1}, u_{2}, u_{3}}(x)=\{(s, t): & s \in 2 \mathbb{N}, t \in \mathbb{N}, t>s+3, d_{t}(x)=v, \\
& \left.d_{s-i}(x)=u_{i} \text { for } i \in\{0,1,2,3\} \text { and } d_{i}(x)=0 \text { for } s<i<t\right\} .
\end{aligned}
$$

Thus $G_{v, u_{0}, u_{1}, u_{2}, u_{3}}(x)$ is the set of "gaps" of the form $v 0 \ldots 0 u_{0} u_{1} u_{2} u_{3}$ with $u_{0}$ in even position and at least three 0 's between $v$ and $u_{0}$, occurring in the base $-p$ expansion of $x$ written with the most significant digit on the left. Define $\psi_{v, u_{0}, u_{1}, u_{2}, u_{3}}(x) \in\{0,1, \ldots, p-1\}$ by

$$
\psi_{v, u_{0}, u_{1}, u_{2}, u_{3}}(x) \equiv\left|G_{v, u_{0}, u_{1}, u_{2}, u_{3}}(x)\right|(\bmod p) .
$$

Let $\theta$ be a finite colouring of $\mathbb{N}$ such that one colour class is $\left\{1,2, \ldots, p^{4}\right\}$ and for $x, y \in \mathbb{N} \backslash\left\{1,2, \ldots, p^{4}\right\}, \theta(x)=\theta(y)$ if and only if

(1) $\phi(x)=\phi(y)$;

(2) $f(x)=f(y)$; and

(3) for all $\left\langle v, u_{0}, u_{1}, u_{2}, u_{3}\right\rangle \in\{1,2, \ldots, p-1\}^{2} \times\{0,1, \ldots, p-1\}^{3}$ and all $i \in\{1,2, \ldots, k\}, \psi_{v, u_{0}, u_{1}, u_{2}, u_{3}}\left(a_{i} x\right)=\psi_{v, u_{0}, u_{1}, u_{2}, u_{3}}\left(a_{i} y\right)$.

Pick $\vec{x} \in \mathbb{N}^{\omega}$ such that $B \vec{x}$ is monochromatic with respect to $\theta$ and for all $t, s \in \omega$, if $p^{s} \leqslant x_{t}$, then $p^{s+8}$ divides $x_{t+1}$.

We note that for all $i, j \in\{1,2, \ldots, k\}$ and all $t<\omega, \max \operatorname{supp}\left(a_{i} x_{t}\right)+3<$ $\min \operatorname{supp}\left(a_{j} x_{t+1}\right)$. To see this, let $s=\max \operatorname{supp}\left(x_{t}\right)$. Then by Lemma 4.4(1), $x_{t}>$ $p^{s-2}$ so $p^{s+6}$ divides $x_{t+1}$, and thus $\min \operatorname{supp}\left(a_{j} x_{t+1}\right)=\min \operatorname{supp}\left(x_{t+1}\right)>s+5 \geqslant$ $\max \operatorname{supp}\left(a_{i} x_{t}\right)+3$, where the last inequality holds by Lemma 4.4(2) or (3).

Let $\left\langle u_{0}, u_{1}, u_{2}, u_{3}\right\rangle=\phi\left(x_{0}\right)$, the constant value of $\phi$ on the entries of $B \vec{x}$. Let $v=$ $f\left(a_{r} x_{0}\right)$. (If $w$ is the constant value of $f$ on the entries of $B \vec{x}$, then $v \equiv a_{r} w(\bmod p)$.) Let $\psi=\psi_{v, u_{0}, u_{1}, u_{2}, u_{3}}$.

Before completing the proof of Theorem 4.5, we establish two lemmas.

Lemma 4.6. Let $x \in \mathbb{N}$ with $x>p^{4}$ and assume that $\phi(x)=\left\langle u_{0}, u_{1}, u_{2}, u_{3}\right\rangle$. If $a \in \mathbb{N}$ with $1<a<\frac{p}{2}$, then $\phi(a x) \neq\left\langle u_{0}, u_{1}, u_{2}, u_{3}\right\rangle$. 
Proof. Suppose that $\phi(a x)=\left\langle u_{0}, u_{1}, u_{2}, u_{3}\right\rangle$. Then the four most significant digits in the base $-p$ expansion of $x$ and $a x$ are the same so there exists $m \in \omega$ such that $\max \operatorname{supp}\left(p^{2 m} x\right)=\max \operatorname{supp}(a x)=s$, say. So we have $p^{2 m} x=y+z$ and $a x=w+z$ for some $y, z, w \in \mathbb{Z}$ satisfying $\max \operatorname{supp}(y) \leqslant s-4$ and $\max \operatorname{supp}(w) \leqslant s-4$. It follows from Lemma 4.4(1) that $\left|p^{2 m}-a\right| x<2 p^{s-3}$. Since $\left|p^{2 m}-a\right| \geqslant 1$ we have that $x<2 p^{s-3}$ so that $a x<p^{s-2}$, contradicting Lemma 4.4(1).

Lemma 4.7. Let $x \in \mathbb{N}$ with $x>p^{4}$, let $y \in \mathbb{Z} \backslash\{0\}$ such that $\max \operatorname{supp}(x)+5<$ $\min \operatorname{supp}(y)$, and let $i \in\{1,2, \ldots, k\}$. If $\phi(x)=\left\langle u_{0}, u_{1}, u_{2}, u_{3}\right\rangle$, then $\bmod p$

$$
\psi\left(a_{i} x+y\right) \equiv \begin{cases}\psi\left(a_{i} x\right)+\psi(y)+1 & \text { if } a_{i}=1 \text { and } f(y)=v \\ \psi\left(a_{i} x\right)+\psi(y) & \text { otherwise }\end{cases}
$$

Proof. $G_{v, u_{0}, u_{1}, u_{2}, u_{3}}\left(a_{i} x+y\right)=G_{v, u_{0}, u_{1}, u_{2}, u_{3}}\left(a_{i} x\right) \cup G_{v, u_{0}, u_{1}, u_{2}, u_{3}}(y) \cup H$, where

$H=\left\{\left(\max \operatorname{supp}\left(a_{i} x\right), \min \operatorname{supp}(y)\right)\right\}$ if $a_{i} x>0, \phi\left(a_{i} x\right)=\left\langle u_{0}, u_{1}, u_{2}, u_{3}\right\rangle$, and $f(y)=v$,

and $H=\emptyset$ otherwise. If $a_{i}<0$, then $a_{i} x<0$, and by Lemma 4.6, if $a_{i}>1$, then $\phi\left(a_{i} x\right) \neq\left\langle u_{0}, u_{1}, u_{2}, u_{3}\right\rangle$.

We are now ready to complete the proof of the theorem.

Since $f\left(x_{0}\right)=f\left(a_{1} x_{j(1)}+a_{2} x_{j(2)}+\ldots+a_{k} x_{j(k)}\right)=f\left(a_{1} x_{j(1)}\right)$, we have that $a_{1}=1$ so $r>1$.

Let $i \in\{1,2, \ldots, k\}$ and $j<\omega$ be given. Then $\psi\left(a_{i} x_{j}+a_{i} x_{j+1}\right)=\psi\left(a_{i} x_{j}\right)=\psi\left(a_{i} x_{j+1}\right)$ and $\phi\left(x_{j}\right)=\left\langle u_{0}, u_{1}, u_{2}, u_{3}\right\rangle$, since $x_{j}, x_{j+1}$ and $x_{j}+x_{j+1}$ are all entries of $B \vec{x}$. We claim that $\psi\left(a_{i} x_{j}+a_{i} x_{j+1}\right)=\psi\left(a_{i} x_{j}\right)+\psi\left(a_{i} x_{j+1}\right)$ so that $\psi\left(a_{i} x_{j}\right)=0$. If not, then by Lemma 4.7, $a_{i}=1$ and $f\left(a_{i} x_{j+1}\right)=v$. But the fact that $f\left(a_{i} x_{j+1}\right)=v=f\left(a_{r} x_{j+1}\right)$ implies that $a_{i}=a_{r} \neq 1$, a contradiction.

By repeated applications of Lemma 4.7 , beginning with $\psi\left(a_{k-1} x_{j(k-1)}+a_{k} x_{j(k)}\right)$, we see that $\psi\left(a_{1} x_{j(1)}+a_{2} x_{j(2)}+\ldots+a_{k} x_{j(k)}\right)$ is the number of $i \in\{1,2, \ldots, k-1\}$ for which $a_{i}=1$ and $a_{i+1}=a_{r}$. Since this number is at least 1 and less than $p$, we have that $\psi\left(a_{1} x_{j(1)}+a_{2} x_{j(2)}+\ldots+a_{k} x_{j(k)}\right) \neq \psi\left(x_{0}\right)$, a contradiction.

\section{Image domination and image maximality}

We shall say that a matrix $A$ is image maximal provided that whenever $B$ is an IPR matrix extending $A$, that is $B$ consists of $A$ with some rows added, then $A$ image dominates $B$.

We note that the Finite Sums matrix $\mathbf{F}$ is not image maximal. Indeed, Let $B$ be $\mathbf{F}$ with the row $\left(\begin{array}{ccccc}1 & 2 & 0 & 0 & \ldots\end{array}\right)$ added. By Theorem 3.4, $B$ is IPR because $\left(\begin{array}{cc}1 & 2 \\ \mathbf{F}_{2}\end{array}\right)$ is a first entries matrix. For $n \in \omega$, let $x_{n}=2^{2 n}$. Then $F S\left(\left\langle 2^{2 n}\right\rangle_{n=0}^{\infty}\right)=\operatorname{Im}(\mathbf{F} \vec{x})$ and $F S\left(\left\langle 2^{2 n}\right\rangle_{n=0}^{\infty}\right)$ contains no image of $B$. (One cannot have $\left\{y_{0}, y_{1}, y_{0}+y_{1}, y_{0}+2 y_{1}\right\} \subseteq F S\left(\left\langle 2^{2 n}\right\rangle_{n=0}^{\infty}\right)$.)

We show now that the DH-matrix $\mathbf{D}$ is finitely image maximal in the sense that any IPR extension of $\mathbf{D}$ obtained by adding finitely many rows is image dominated by $\mathbf{D}$. 
Theorem 5.1. Let $m \in \mathbb{N}$ and let $C$ be an $m \times \omega$ matrix such that $A=\left(\begin{array}{c}C \\ \mathbf{D}\end{array}\right)$ is IPR. Then $\mathbf{D}$ image dominates $A$.

Proof. Let $\left\langle B_{n}\right\rangle_{n=0}^{\infty},\langle u(n)\rangle_{n=0}^{\infty}$, and $\langle v(n)\rangle_{n=0}^{\infty}$, be as in the construction of $\mathbf{D}$. Define $k(0)=0$ and for each $n<\omega$, let $k(n+1)=k(n)+v(n)$. (Then any row of $\mathbf{D}$ has in columns $k(n), k(n)+1, \ldots, k(n)+v(n)-1$ either all 0 's or a row of $B_{n}$.)

Pick $\delta \in \mathbb{N}$ such that for all $i \in\{0,1, \ldots, m-1\}$ and all $j \geqslant k(\delta), c_{i, j}=0$. Let $N$ be the restriction of $A$ to columns $0,1, \ldots, k(\delta)-1$. Let $M$ be a finite matrix whose rows are the nonzero rows of $N$ without repetition. Then $M$ is a finite IPR matrix since each row of $M$ followed by all 0's is a row of $A$. So $M=B_{l}$ for some $l \in \omega$. Note that $v(l)=k(\delta)$.

Choose an injective $f:\{\delta, \delta+1, \ldots\} \rightarrow \mathbb{N} \backslash\{0\}$ so that for each $n \geqslant \delta$, the rows of $B_{n}$ are contained in the rows of $B_{f(n)}$ and $v(f(n))=v(n)$.

Now let $\vec{x} \in \mathbb{N}^{\omega}$. We shall define $\vec{y}$ so that the set of entries of $A \vec{y}$ is contained in the set of entries of $\mathbf{D} \vec{x}$. For $i \in\{0,1, \ldots, k(\delta)-1\}$, let $y_{i}=x_{k(l)+i}$. For $n \geqslant \delta$ and $i \in\{0,1, \ldots, v(n)-1\}$, let $y_{k(n)+i}=x_{k(f(n))+i}$.

To see that the set of entries of $A \vec{y}$ are contained in the set of entries of $\mathbf{D} \vec{x}$, let $\vec{r}$ be a row of $A$. Define a row $\vec{s}$ of $\mathbf{D}$ as follows. For $i \in\{0,1, \ldots, k(\delta)-1\}$, let $s_{k(l)+i}=r_{i}$. For $n \geqslant \delta$ and $i \in\{0,1, \ldots, v(n)-1\}$, let $s_{k(f(n))+i}=r_{k(n)+i}$. If $n \in \omega \backslash(f[\{\delta, \delta+1, \ldots\}] \cup\{l\})$ and $i \in\{0,1, \ldots, v(n)-1\}$, then $s_{k(n)+i}=0$. Then $\vec{r} \cdot \vec{y}=\vec{s} \cdot \vec{x}$.

Conjecture 5.2. The system $\mathbf{D}$ is image maximal.

The DH-matrix $\mathbf{D}$ seems a good candidate for a universal centrally IPR matrix. It trivially image dominates any finite IPR matrix. By Theorem 2.6 it is strongly centrally IPR. Therefore, if $\mathbf{D}$ image dominates a matrix $A$, it is immediate that $A$ is centrally IPR. We see now, however, that $A$ need not be strongly centrally IPR.

Theorem 5.3. Let $A$ be any strongly centrally IPR matrix and let

$$
B=\left(\begin{array}{cc}
1 & 0 \\
3 & -1 \\
5 & -2 \\
\vdots & \vdots
\end{array}\right)
$$

Then $B$ is not strongly centrally IPR and $A$ image dominates $B$.

Proof. By [8, Theorem 2.11] $B$ is not strongly centrally IPR. To see that $A$ image dominates $B$, let $a$ be any element of an image of $A$. Let $y_{0}=a$ and $y_{1}=2 a$. Then $\operatorname{Im}(B \vec{y})=\{a\}$.

One might hope (and we did) that any centrally IPR matrix is image dominated by $\mathbf{D}$, or at least that any strongly centrally IPR matrix is image dominated by $\mathbf{D}$. (We knew that no Milliken-Taylor matrix which is not essentially a multiple of $\mathbf{F}$ is image dominated by D.) We shall see that this fails. To see it, we shall need another version of a DH-matrix (which is closer to the original in [4]). The next definition differs from the description in Section 2 in that here the entries are required to be non negative. 
Definition 5.4. Let $(m, p, c) \in \mathbb{N}^{3}$. A matrix $A$ is an $(m, p, c)$-matrix if and only if $A$ is a first entries matrix with $m$ columns, all first entries are equal to $c$, all entries of $A$ are in $\{0,1, \ldots, p\}$, and $A$ contains all rows possible subject to these restrictions.

Lemma 5.5. Let $u, v \in \mathbb{N}$ and let $A$ be $a u \times v$ matrix with entries from $\mathbb{Q}$. Then $A$ is $I P R$ if and only if there exist $(m, p, c) \in \mathbb{N}^{3}$ such that for all $p^{\prime} \geqslant p$, every $\left(m, p^{\prime}, c\right)$-matrix $B$, and every $\vec{y} \in \mathbb{N}^{m}$, there exists $\vec{x} \in \mathbb{N}^{v}$ such that $\operatorname{Im}(A \vec{x}) \subseteq \operatorname{Im}(B \vec{y})$.

Proof. Since $(m, p, c)$-matrices are first entries matrices, the sufficiency is immediate. So assume that $A$ is IPR. Pick by Theorem 2.2(c) $m \in \mathbb{N}$, a $u \times m$ matrix $E$ with entries from $\omega$, and $c \in \mathbb{N}$ such that $E$ satisfies the first entries condition, $c$ is the only first entry of $E$, and given any $\vec{y} \in \mathbb{N}^{m}$ there is some $\vec{x} \in \mathbb{N}^{v}$ with $A \vec{x}=E \vec{y}$. Let $p$ be the maximum of all of the entries of $E$, let $p^{\prime} \geqslant p$, and let $B$ be an $\left(m, p^{\prime}, c\right)$-matrix. Let $\vec{y} \in \mathbb{N}^{m}$ be given and pick $\vec{x} \in \mathbb{N}^{v}$ such that $A \vec{x}=E \vec{y}$. Then $\operatorname{Im}(A \vec{x})=\operatorname{Im}(E \vec{y}) \subseteq \operatorname{Im}(B \vec{y})$.

Now we define our second version of a DH-matrix. First fix an enumeration $\left\langle B_{n}^{\prime}\right\rangle_{n=0}^{\infty}$ of the $(m, p, c)$-matrices where each $B_{n}^{\prime}$ is an $(m(n), p(n), c(n))$-matrix. For each $i \in \mathbb{N}$, let $\overrightarrow{0}_{i}$ be the 0 vector with $i$ entries. Let $\mathbf{D}^{\prime}$ be an $\omega \times \omega$ matrix with all rows of the form $\vec{r}_{0} \frown \vec{r}_{1} \frown \vec{r}_{2} \frown \ldots$ where each $\vec{r}_{i}$ is either $\overrightarrow{0}_{m(i)}$ or is a row of $B_{i}^{\prime}$, and all but finitely many are $\overrightarrow{0}_{m(i)}$.

Theorem 5.6. The DH-matrices $\mathbf{D}$ and $\mathbf{D}^{\prime}$ are image equivalent. That is, each image dominates the other.

Proof. Let $\left\langle B_{n}\right\rangle_{n=0}^{\infty},\langle v(n)\rangle_{n=0}^{\infty},\left\langle B_{n}^{\prime}\right\rangle_{n=0}^{\infty}$, and $\langle m(n)\rangle_{n=0}^{\infty}$ be as in the construction of $\mathbf{D}$ and $\mathbf{D}^{\prime}$. Since each $B_{n}^{\prime}$ is some $B_{k}$, the fact that $\mathbf{D}$ image dominates $\mathbf{D}^{\prime}$ is immediate.

We now show that $\mathbf{D}^{\prime}$ image dominates $\mathbf{D}$. Using Lemma 5.5, inductively define an injection $f: \omega \rightarrow \omega$ such that for every $\vec{y} \in \mathbb{N}^{m(f(n))}$, there exists $\vec{x} \in \mathbb{N}^{v(n)}$ such that $\operatorname{Im}\left(B_{n} \vec{x}\right) \subseteq \operatorname{Im}\left(B_{f(n)}^{\prime} \vec{y}\right)$.

Inductively define $k(n)$ and $l(n)$ for $n \in \omega$ by $k(0)=l(0)=0$, and for $n \in \omega$, $k(n+1)=k(n)+v(n)$ and $l(n+1)=l(n)+m(n)$. To see that $\mathbf{D}^{\prime}$ image dominates $\mathbf{D}$, let $\vec{w} \in \mathbb{N}^{\omega}$ be given. For $n \in \omega$, define $\vec{y}_{n} \in \mathbb{N}^{m(f(n))}$ by, for $i \in\{0,1, \ldots, m(f(n))-1\}$, $y_{n, i}=w_{l(f(n))+i}$, and pick $\vec{x}_{n} \in \mathbb{N}^{v(n)}$ such that $\operatorname{Im}\left(B_{n} \vec{x}_{n}\right) \subseteq \operatorname{Im}\left(B_{f(n)}^{\prime} \vec{y}_{n}\right)$. Define $\vec{z} \in \mathbb{N}^{\omega}$ by, for $n \in \omega$ and $i \in\{0,1, \ldots, v(n)-1\}, z_{k(n)+i}=x_{n, i}$. Then as in the proof of Theorem 5.1 , one sees that $\operatorname{Im}(\mathbf{D} \vec{z}) \subseteq \operatorname{Im}\left(\mathbf{D}^{\prime} \vec{w}\right)$.

Let $\left\langle c_{n}\right\rangle_{n=1}^{\infty}$ be a sequence in $\mathbb{N}$ and let $\mathcal{I}$ be the matrix defined in Figure 5 . As in [1, Theorem 16], one can show that $\mathcal{I}$ is IPR. One can in fact show that it is strongly centrally IPR. One can also show that if the sequence $\left\langle c_{n}\right\rangle_{n=1}^{\infty}$ is unbounded, then $\mathbf{D}$ does not image dominate $\mathcal{I}$. We omit the verification of these assertions because we have a much stronger example.

Theorem 5.7. There is an $(\omega+\omega) \times \omega$ matrix $C$ with all entries from $\{0,1,2\}$ and all column sums equal to 3 or 4 which is strongly centrally IPR but is not image dominated by $\mathbf{D}$. 


$$
\mathcal{I}=\left(\begin{array}{ccccccccc}
1 & 0 & 0 & 0 & 0 & 0 & 0 & 0 & \ldots \\
0 & 1 & 0 & 0 & 0 & 0 & 0 & 0 & \ldots \\
c_{1} & 1 & 0 & 0 & 0 & 0 & 0 & 0 & \ldots \\
0 & 0 & 1 & 0 & 0 & 0 & 0 & 0 & \ldots \\
0 & 0 & 0 & 1 & 0 & 0 & 0 & 0 & \ldots \\
c_{2} & 0 & 1 & 1 & 0 & 0 & 0 & 0 & \ldots \\
0 & 0 & 0 & 0 & 1 & 0 & 0 & 0 & \ldots \\
0 & 0 & 0 & 0 & 0 & 1 & 0 & 0 & \ldots \\
0 & 0 & 0 & 0 & 0 & 0 & 1 & 0 & \ldots \\
c_{3} & 0 & 0 & 0 & 1 & 1 & 1 & 0 & \ldots \\
\vdots & \vdots & \vdots & \vdots & \vdots & \vdots & \vdots & \vdots & \ddots
\end{array}\right)
$$

Figure 1: The matrix $\mathcal{I}$.

Proof. Let $A$ be the $\omega \times \omega$ matrix such that, for $i, j \in \omega$,

$$
a_{i, j}= \begin{cases}0 & \text { if } j<i \\ 2 & \text { if } j=i \\ 0 & \text { if } i<j<2^{i} \\ 1 & \text { if } 2^{i} \leqslant j<2^{i+1} \\ 0 & \text { if } 2^{i+1} \leqslant j\end{cases}
$$

so that

$$
A=\left(\begin{array}{cccccccccc}
2 & 1 & 0 & 0 & 0 & 0 & 0 & 0 & 0 & \ldots \\
0 & 2 & 1 & 1 & 0 & 0 & 0 & 0 & 0 & \ldots \\
0 & 0 & 2 & 0 & 1 & 1 & 1 & 1 & 0 & \ldots \\
\vdots & \vdots & \vdots & \vdots & \vdots & \vdots & \vdots & \vdots & \vdots & \ddots
\end{array}\right)
$$

Let $I$ be the $\omega \times \omega$ identity matrix and let $C=\left(\begin{array}{c}I \\ A\end{array}\right)$. By [2, Corollary 3.8] $C$ is strongly centrally IPR. We shall show that $C$ is not image dominated by $\mathbf{D}$ for which it suffices in view of Theorem 5.6 to show that $C$ is not image dominated by $\mathbf{D}^{\prime}$. Let $\left\langle B_{n}^{\prime}\right\rangle_{n=0}^{\infty}$ be as in the construction of $\mathbf{D}^{\prime}$ where each $B_{n}^{\prime}$ is an $(m(n), p(n), c(n))$-matrix.

Define a function $f$ on $\omega$ by $f(0)=1$ and $f(i+1)=2^{f(i)+1}-1$. We will define $\left\langle x_{i, j}\right\rangle_{j=0}^{m(i)-1}$ by induction on $i$.

When we have defined $\left\langle x_{i, j}\right\rangle_{j=0}^{m(i)-1}$, we will let $S_{i}$ be the set of entries of

$$
B_{i}^{\prime}\left(\begin{array}{c}
x_{i, 0} \\
\vdots \\
x_{i, m(i)-1}
\end{array}\right)
$$


and let $M_{i}=\max \left\{S_{0}+S_{1}+\ldots+S_{i}\right\}$.

Pick $b_{0}>\max \left\{\left(2+2^{f(m(0))}\right) c(0), p(0)\right\}$ and for $j \in\{0,1, \ldots, m(0)-1\}$, let $x_{0, j}=b_{0}^{j+1}$. Let $i>0$ and assume we have chosen $\left\langle x_{i-1, j}\right\rangle_{j=0}^{m(i-1)-1}, S_{i-1}$, and $M_{i-1}$. Pick

$$
b_{i}>\max \left\{\left(2+2^{f(m(i))}\right) c(i),\left(2+2^{f(m(i))}\right) M_{i-1}, p(i)\right\}
$$

such that $b_{i-1}$ divides $b_{i}$. For $j \in\{0,1, \ldots, m(i)-1\}$, let $x_{i, j}=b_{i}^{j+1}$.

Notice that since $b_{i}>p(i)$ we have that expressions in

$$
B_{i}\left(\begin{array}{c}
x_{i, 0} \\
\vdots \\
x_{i, m(i)-1}
\end{array}\right)
$$

are unique. That is, if $\vec{r}$ and $\vec{s}$ are rows of $B_{i}$ and

$$
\vec{r}\left(\begin{array}{c}
x_{i, 0} \\
\vdots \\
x_{i, m(i)-1}
\end{array}\right)=\vec{s}\left(\begin{array}{c}
x_{i, 0} \\
\vdots \\
x_{i, m(i)-1}
\end{array}\right),
$$

then $\vec{r}=\vec{s}$.

Notice that, given $y \in S_{i}$, there exist some $l \in\{1,2, \ldots, m(i)\}$ and some $d \in \omega$ such that $y=c(i) b_{i}^{l}+d b_{i}^{l+1}$.

The construction being complete, let

$$
\vec{z}=\left(\begin{array}{c}
x_{0,0} \\
\vdots \\
x_{0, m(0)-1} \\
x_{1,0} \\
\vdots \\
x_{1, m(1)-1} \\
\vdots
\end{array}\right)
$$

and let $J=\operatorname{Im}\left(\mathbf{D}^{\prime} \vec{z}\right)$. Notice that $J=\bigcup_{F \in \mathcal{P}_{f}(\omega)} \sum_{i \in F} S_{i}=F S\left(\left\langle S_{i}\right\rangle_{i=0}^{\infty}\right)$.

For $y \in J$, we let $\operatorname{Supp}(y)$ be that $F \in \mathcal{P}_{f}(\omega)$ such that $y \in \sum_{i \in F} S_{i}$. And for $y \in J$ and $i \in \mathbb{N}$, we let $\pi_{i}(y)=0$ if $i \notin \operatorname{Supp}(y)$ and otherwise, let $\pi_{i}(y) \in S_{i}$ be such that $y=\sum_{i \in \operatorname{Supp}(y)} \pi_{i}(y)$.

Given $i \in \omega$, we have that two elements of $S_{i+1}$ differ by at least $b_{i+1}$ and $b_{i+1}>M_{i}$ so expressions in $\sum_{i \in \operatorname{Supp}(y)} S_{i}$ are unique and thus $\pi_{i}$ is well defined.

We claim that there is no $\vec{y} \in \mathbb{N}^{\omega}$ such that $\operatorname{Im}(C \vec{y}) \subseteq J$, so suppose instead that we have such $\vec{y}$. Let $q=\min \operatorname{Supp}\left(y_{0}\right)$. (Any other member of $\operatorname{Supp}\left(y_{0}\right)$ would do just as well, with no change in the proof.)

Before completing the proof of Theorem 5.7, we establish two lemmas. In the first of these lemmas, note that $2 y_{v}+\sum_{k=2^{v}}^{2^{v+1}} y_{k}$ is entry $v$ of $A \vec{y}$. The point of the proof is that $b_{i+1}$ divides every entry of $S_{i}$ for every $i>q$. 
Lemma 5.8. Let $v \in\{0,1, \ldots, f(m(q))\}$. Then

$$
\pi_{q}\left(2 y_{v}+\sum_{k=2^{v}}^{2^{v+1}-1} y_{k}\right)=2 \pi_{q}\left(y_{v}\right)+\sum_{k=2^{v}}^{2^{v+1}-1} \pi_{q}\left(y_{k}\right)
$$

Proof. Let $z=2 y_{v}+\sum_{k=2^{v}}^{2^{v+1}-1} y_{k}$. Then $z \in J$ so pick $a_{0} \in \omega$ such that $z=a_{0} b_{q+1}+$ $\pi_{q}(z)+\sum_{i=0}^{q-1} \pi_{i}(z)$. (Here $a_{0} b_{q+1}=\sum\left\{\pi_{i}(z): i \in \operatorname{Supp}(z)\right.$ and $\left.i>q\right\}$.)

For each $k \in\{v\} \cup\left\{2^{v}, 2^{v}+1, \ldots, 2^{v+1}-1\right\}$, pick $a_{k} \in \omega$ such that $y_{k}=a_{k} b_{q+1}+$ $\pi_{q}\left(y_{k}\right)+\sum_{i=0}^{q-1} \pi_{i}\left(y_{k}\right)$. Then

$$
\begin{aligned}
z=\left(2 a_{v}\right. & \left.+\sum_{k=2^{v}}^{2^{v+1}-1} a_{k}\right) b_{q+1}+2 \pi_{q}\left(y_{v}\right)+\sum_{k=2^{v}}^{2^{v+1}-1} \pi_{q}\left(y_{k}\right) \\
& +\sum_{i=0}^{q-1}\left(2 \pi_{i}\left(y_{v}\right)+\sum_{k=2^{v}}^{2^{v+1}-1} \pi_{i}\left(y_{k}\right)\right) .
\end{aligned}
$$

Now $\sum_{i=0}^{q-1}\left(2 \pi_{i}\left(y_{v}\right)+\sum_{k=2^{v}}^{2^{v+1}-1} \pi_{i}\left(y_{k}\right)\right) \leqslant\left(2^{v}+2\right) M_{q-1} \leqslant\left(2^{f(m(q))}+2\right) M_{q-1}<b_{q}$. And of course $\sum_{i=0}^{q-1} \pi_{i}(z) \leqslant M_{q-1}<b_{q}$.

Since also $b_{q}$ divides $a_{0} b_{q+1}+\pi_{q}(z)$ and $b_{q}$ divides

$$
\left(2 a_{v}+\sum_{k=2^{v}}^{2^{v+1}-1} a_{k}\right) b_{q+1}+2 \pi_{q}\left(y_{v}\right)+\sum_{k=2^{v}}^{2^{v+1}-1} \pi_{q}\left(y_{k}\right)
$$

we have that

$$
\left(2 a_{v}+\sum_{k=2^{v}}^{2^{v+1}-1} a_{k}\right) b_{q+1}+2 \pi_{q}\left(y_{v}\right)+\sum_{k=2^{v}}^{2^{v+1}-1} \pi_{q}\left(y_{k}\right)=a_{0} b_{q+1}+\pi_{q}(z) .
$$

Similarly $2 \pi_{q}\left(y_{v}\right)+\sum_{k=2^{v}}^{2^{v+1}-1} \pi_{q}\left(y_{k}\right)<b_{q+1}$ and $\pi_{q}(z)<b_{q+1}$ so these are equal as claimed.

Lemma 5.9. Let $v \in\{0,1, \ldots, f(m(q))\}$ such that $\pi_{q}\left(y_{v}\right) \neq 0$. Pick $l \in\{1,2, \ldots, m(q)\}$ and $d \in \omega$ such that $\pi_{q}\left(y_{v}\right)=c(q) b^{l}+d b_{q}^{l+1}$. Then $l \geqslant 2$ and for some $i \in\left\{2^{v}, 2^{v}+\right.$ $\left.1, \ldots, 2^{v+1}-1\right\}$, some $l^{\prime} \in\{1,2, \ldots, l-1\}$, and some $d^{\prime} \in \omega, \pi_{q}\left(y_{i}\right)=c(q) b^{l^{\prime}}+d^{\prime} b_{q}^{l^{\prime}+1}$.

Proof. Since $\pi_{q}\left(y_{v}\right) \neq 0$ we have by Lemma 5.8 that $\pi_{q}\left(2 y_{v}+\sum_{k=2^{v}}^{2^{v+1}} y_{k}\right) \neq 0$. Pick $t \in\{1,2, \ldots, m(q)\}$ and $e \in \omega$ such that $\pi_{q}\left(2 y_{v}+\sum_{k=2^{v}}^{2^{v+1}-1} y_{k}\right)=c(q) b_{q}^{t}+e b_{q}^{t+1}$.

Let $H=\left\{k \in\left\{2^{v}, 2^{v}+1, \ldots, 2^{v+1}-1\right\}: \pi_{q}\left(y_{k}\right) \neq 0\right\}$. If $H=\emptyset$, then by Lemma 5.8, $c(q) b_{q}^{t}+e b_{q}^{t+1}=2 c(q) b_{q}^{l}+2 d b_{q}^{l+1}$ so, since $b_{q}>2 c(q)$, we have $t=l$ and $c(q)=2 c(q)$, a contradiction. So $H \neq \emptyset$.

For $k \in H$, pick $l_{k} \in\{1,2, \ldots, m(q)\}$ and $d_{k} \in \omega$ such that $\pi_{q}\left(y_{k}\right)=c(q) b_{q}^{l_{k}}+d_{k} b_{q}^{l_{k}+1}$. We need to show that some $l_{k}<l$, so suppose instead that each $l_{k} \geqslant l$. We have by Lemma 5.8 that

$$
c(q) b_{q}^{t}+e b_{q}^{t+1}=2 c(q) b_{q}^{l}+2 d b_{q}^{l+1}+\sum_{k \in H}\left(c(q) b_{q}^{l_{k}}+d_{k} b_{q}^{l_{k}+1}\right) .
$$

If each $l_{k}>l$ we again conclude that $t=l$ and $c(q)=2 c(q)$. Let $K=\left\{k \in H: l_{k}=l\right\}$ and let $\delta=|K|$. Then we get

$$
2 c(q) b_{q}^{l}+2 d b_{q}^{l+1}+\sum_{k \in H}\left(c(q) b_{q}^{l_{k}}+d_{k} b_{q}^{l_{k}+1}\right)=(2+\delta) c(q) b_{q}^{l}+\alpha b_{q}^{l+1}
$$

for some $\alpha \in \omega$. But $\delta \leqslant 2^{v} \leqslant 2^{f(m(q))}$ so $(2+\delta) c(q) \leqslant\left(2+2^{f(m(q))}\right) c(q)<b_{q}$ so $t=l$ and $c(q)=(2+\delta) c(q)$, a contradiction. 
We are now ready to complete the proof of the theorem. Pick $l_{0} \in\{1,2, \ldots, m(q)\}$ and $d_{0} \in \omega$ such that $\pi_{q}\left(y_{0}\right)=c(q) b_{q}^{l_{0}}+d_{0} b_{q}^{l_{0}+1}$. By Lemma 5.9, $l_{0} \geqslant 2$ and we may pick $i(1)=1, d_{1} \in \omega$, and $l_{1} \in\left\{1,2, \ldots, l_{0}-1\right\}$ such that $\pi_{q}\left(y_{i(1)}\right)=c(q) b_{q}^{l_{1}}+d_{1} b_{q}^{l_{1}+1}$.

Given $t \geqslant 1, i(t), l_{t}$, and $d_{t}$ such that $i(t) \leqslant f(t) \leqslant f(m(q))$ and $\pi_{q}\left(y_{i(t)}\right)=c(q) b_{q}^{l_{t}}+$ $d_{t} b_{q}^{l_{t}+1}$, pick by Lemma 5.9, $i(t+1) \in\left\{2^{i(t)}, 2^{i(t)}+1, \ldots, 2^{i(t)+1}-1\right\}, l_{t+1}<l_{t}$, and $d_{t+1} \in \omega$ such that $\pi_{q}\left(y_{i(t+1)}\right)=c(q) b_{q}^{l_{t+1}}+d_{t+1} b_{q}^{l_{t+1}+1}$. Then $i(t+1) \leqslant 2^{i(t)+1}-1 \leqslant$ $2^{f(t)+1}-1=f(t+1)$. Also $m(q) \geqslant l_{0}>l_{1}>\ldots>l_{t+1}$ so $m(q)>t+1$ and thus $i(t+1) \leqslant f(t+1)<f(m(q))$. When $t+1=m(q)$ we have a contradiction.

Note that the matrix of Theorem 5.7 has unbounded row sums (as does $\mathbf{D}^{\prime}$ ).

Question 5.10. Let $A$ be an $\omega \times \omega$ centrally IPR matrix with the property that $\left\{\sum_{j=0}^{\infty}\left|a_{i, j}\right|: i<\omega\right\}$ is bounded. Must $A$ be image dominated by $\mathbf{D}$ ?

\section{Translates of MT-Matrices}

As we saw in Theorem 1.5, if $k \in \mathbb{N}, \vec{a}=\left\langle a_{0}, a_{1}, \ldots, a_{k}\right\rangle$ is a compressed sequence, and $M$ is an $M T(\vec{a})$ matrix, then $\left(\begin{array}{cc}M & \mathbf{O} \\ \mathbf{O} & \mathbf{F}\end{array}\right)$ is not IPR. We shall see in Theorem 6.3 , that if $a_{k}=1$, then $\left(\begin{array}{cc}\overline{1} & M \\ \overline{0} & \mathbf{F}\end{array}\right)$ is partition regular, where $\overline{1}$ and $\overline{0}$ are the constant length $\omega$ column vectors. That is, given any finite colouring of $\mathbb{N}$, there must exist a sequence $\vec{x}=\left\langle x_{n}\right\rangle_{n=0}^{\infty}$ and $b \in \mathbb{N}$ such that $F S(\vec{x}) \cup(b+M T(\vec{a}, \vec{x}))$ is monochromatic.

Given $a \in \mathbb{Z}$ and $p \in \beta \mathbb{N}$, by ap we mean the product in $(\beta \mathbb{Z}, \cdot)$. (If $p \in \mathbb{N}^{*}$ it is not even true that $2 p=p+p$.) If $A \subseteq \mathbb{Z}$, then $A \in a p$ if and only if $a^{-1} A \in p$. Since $\mathbb{N} \in p$, we have that $A \in a p$ if and only if $\{x \in \mathbb{N}: a x \in A\} \in p$.

The basic algebraic property of $\beta \mathbb{N}$ used in the following lemma is that $p+\beta \mathbb{N}+p$ is a group (with identity $p$ ) in $\beta \mathbb{N}$ whenever $p$ is an idempotent in the smallest ideal of $\beta \mathbb{N}$.

Lemma 6.1. Let $k \in \mathbb{N}$ and let $\vec{a}=\left\langle a_{0}, a_{1}, \ldots, a_{k}\right\rangle$ be a compressed sequence in $\mathbb{Z} \backslash\{0\}$ with $a_{k}=1$. Let $p$ be a minimal idempotent in $\beta \mathbb{N}$ and let $A \in p$. There exists $b \in \mathbb{N}$ such that $-b+A \in a_{0} p+a_{1} p+\ldots+a_{k} p$.

Proof. By [10, Exercise 4.3.5], $\mathbb{N}^{*}$ is a left ideal of $(\beta \mathbb{Z},+)$, so $\beta \mathbb{N}+p \subseteq \beta \mathbb{Z}+p=$ $\beta \mathbb{Z}+p+p \subseteq \mathbb{N}^{*}+p \subseteq \beta \mathbb{N}+p$. Therefore

$$
\begin{aligned}
p+a_{0} p+a_{1} p+\ldots+a_{k} p & =p+\left(a_{0} p+\ldots+a_{k-1} p\right)+p \\
& \in p+\beta \mathbb{Z}+p \\
& =p+\beta \mathbb{N}+p
\end{aligned}
$$

and, since $p$ is minimal, $p+\beta \mathbb{N}+p$ is a group. Pick $q \in p+\beta \mathbb{N}+p$ such that $q+p+$ $a_{0} p+a_{1} p+\ldots+a_{k} p=p$. Since $q+p=q, A \in q+a_{0} p+a_{1} p+\ldots+a_{k} p$ so

$$
\left\{x \in \mathbb{N}:-x+A \in a_{0} p+a_{1} p+\ldots+a_{k} p\right\} \in q .
$$

Pick $b \in\left\{x \in \mathbb{N}:-x+A \in a_{0} p+a_{1} p+\ldots+a_{k} p\right\}$. 
Before giving the proof of Theorem 6.3 in the general case, we shall first give the proof for a simple special case. We should like the reader to understand the simple idea underlying the proof, before having to read the rather daunting details of the general proof.

Theorem 6.2. Let $\vec{a}=\langle 2,1\rangle$, let $p$ be a minimal idempotent in $\beta \mathbb{N}$ and let $A \in p$. Then there exist $b \in \mathbb{N}$ and a sequence $\left\langle x_{n}\right\rangle_{n=0}^{\infty}$ in $\mathbb{N}$ such that $F S\left(\left\langle x_{n}\right\rangle_{n=0}^{\infty}\right) \subseteq A$ and $b+M T\left(\vec{a},\left\langle x_{n}\right\rangle_{n=0}^{\infty}\right) \subseteq A$.

Proof. By Lemma 6.1 We can choose $b \in \mathbb{N}$ such that $-b+A \in 2 p+p$. Given $B \in p$, let $B^{\star}=\{x \in B:-x+B \in p\}$. By Lemma 2.3, $B^{\star} \in p$ and, if $x \in B^{\star}$, then $-x+B^{\star} \in p$.

We put $B=\{x \in \mathbb{N}: 2 x+p \in \overline{-b+A}\}$ and, for each $x \in B$, we put

$$
B(x)=\{y \in \mathbb{N}: 2 x+y \in-b+A\} .
$$

We observe that $B$ and $B(x)$ are members of $p$.

We shall inductively construct a sequence $\left\langle x_{n}\right\rangle_{n=0}^{\infty}$ in $\mathbb{N}$ such that $F S\left(\left\langle x_{n}\right\rangle_{n=0}^{\infty}\right) \subseteq$ $A^{\star} \cap B^{\star}$ and, whenever $F, G \in \mathcal{P}_{f}(\mathbb{N})$ and $F<G$, then $\sum_{n \in G} x_{n} \in B\left(\sum_{m \in F} x_{m}\right)^{\star}$.

We choose any $x_{0} \in A^{\star} \cap B^{\star}$. We then assume that $r \geqslant 0$ and that we have chosen a sequence $\left\langle x_{0}, x_{1}, x_{2} \ldots, x_{r}\right\rangle$ so that $F S\left(\left\langle x_{n}\right\rangle_{n=0}^{r}\right) \subseteq A^{\star} \cap B^{\star}$, and, whenever $F, G \in$ $\mathcal{P}_{f}(\{0,1, \ldots, r\})$ and $F<G$, then $\sum_{n \in G} x_{n} \in B\left(\sum_{m \in F} x_{m}\right)^{\star}$.

If $F \in \mathcal{P}_{f}(\{0,1, \ldots, r\})$, then the following sets are all members of $p:-\sum_{m \in F} x_{m}+A^{\star}$, $-\sum_{m \in F} x_{m}+B^{\star}$, and $B\left(\sum_{m \in F} x_{m}\right)^{\star}$. Furthermore, if $G \in \mathcal{P}_{f}(\{1,2, \ldots, r\})$ and $F<G$, then $-\sum_{n \in G} x_{n}+B\left(\sum_{m \in F} x_{m}\right)^{\star} \in p$. So all the sets of this form have a non-empty intersection with $A^{\star} \cap B^{\star}$, and we can choose an element $x_{r+1} \in A^{\star} \cap B^{\star}$ which is in all these sets. It is then routine to check that our inductive hypotheses extend to the sequence $\left\langle x_{0}, x_{1}, \ldots, x_{r}, x_{r+1}\right\rangle$.

Note that, in the following theorem, if one wishes, one can let $\left\langle\vec{a}_{i}\right\rangle_{i=0}^{\infty}$ enumerate all of the compressed sequences in $\mathbb{Z} \backslash\{0\}$ with final term equal to 1 . The proof of the following theorem is based on the proof of [10, Theorem 17.31].

Theorem 6.3. For each $i<\omega$, let $k(i) \in \mathbb{N}$ and let $\vec{a}_{i}=\left\langle a_{i, 0}, a_{i, 1}, \ldots, a_{i, k(i)}\right\rangle$ be a compressed sequence in $\mathbb{Z} \backslash\{0\}$ with $a_{i, k(i)}=1$. Let $p$ be a minimal idempotent in $\beta \mathbb{N}$ and let $A \in p$. There exists sequences $\left\langle b_{n}\right\rangle_{n=0}^{\infty}$ and $\left\langle x_{n}\right\rangle_{n=0}^{\infty}$ in $\mathbb{N}$ such that $F S\left(\left\langle x_{n}\right\rangle_{n=0}^{\infty}\right) \subseteq A$ and for each $i \in \omega, b_{i}+M T\left(\vec{a}_{i},\left\langle x_{n}\right\rangle_{n=i}^{\infty}\right) \subseteq A$.

Proof. For each $i \in \omega$, pick by Lemma $6.1, b_{i} \in \mathbb{N}$ such that

$$
-b_{i}+A \in a_{i, 0} p+a_{i, 1} p+\ldots+a_{i, k(i)} p .
$$

Given $B \in p$, let $B^{\star}=\{x \in B:-x+B \in p\}$. By Lemma 2.3, if $x \in B^{\star}$, then $-x+B^{\star} \in p$.

Let $B_{0}=A \cap\left\{x \in \mathbb{N}:-a_{0,0} x+\left(-b_{0}+A\right) \in a_{0,1} p+\ldots+a_{0, k(0)} p\right\}$, note that $B_{0} \in p$, and pick $x_{0} \in B_{0}^{\star}$.

Now let $n \in \omega$ and assume that we have chosen $\left\langle x_{j}\right\rangle_{j=0}^{n}$ in $\mathbb{N}$ and $\left\langle B_{j}\right\rangle_{j=0}^{n}$ in $p$ so that for each $r \in\{0,1, \ldots, n\}$ the following induction hypotheses hold. 
(I) $B_{r} \subseteq\left\{x \in \mathbb{N}:-a_{r, 0} x+\left(-b_{r}+A\right) \in a_{r, 1} p+\ldots+a_{r, k(r)} p\right\}$.

(II) If $\emptyset \neq F \subseteq\{0,1, \ldots, r\}$ and $i=\min F$, then $\sum_{t \in F} x_{t} \in B_{i}^{\star}$.

(III) If $r<n$, then $B_{r+1} \subseteq B_{r}$.

(IV) If $i \in\{0,1, \ldots, r\}, l \in\{0,1, \ldots, k(i)-1\}, F_{0}, F_{1}, \ldots, F_{l} \in \mathcal{P}_{f}(\{i, i+1, \ldots, r\})$, and $F_{0}<F_{1}<\ldots<F_{l}$, then $-\sum_{j=0}^{l} a_{i, j} \sum_{t \in F_{j}} x_{t}+\left(-b_{i}+A\right) \in a_{i, l+1} p+\ldots+a_{i, k(i)} p$.

(V) If $i \in\{0,1, \ldots, r\}, F_{0}, F_{1}, \ldots, F_{k(i)-1} \in \mathcal{P}_{f}(\{i, i+1, \ldots, r\}), F_{0}<F_{1}<\ldots<F_{k(i)-1}$, and $r<n$, then $B_{r+1} \subseteq-\sum_{j=0}^{k(i)-1} a_{i, j} \sum_{t \in F_{j}} x_{t}+\left(-b_{i}+A\right)$.

(VI) If $i \in\{0,1, \ldots, r\}, l \in\{0,1, \ldots, k(i)-2\}, F_{0}, F_{1}, \ldots, F_{l} \in \mathcal{P}_{f}(\{i, i+1, \ldots, r\})$, $F_{0}<F_{1}<\ldots<F_{l}$, and $r<n$, then

$$
\begin{aligned}
B_{r+1} \subseteq\{x \in \mathbb{N}: & -a_{i, l+1} x+\left(-\sum_{j=0}^{l} a_{i, j} \sum_{t \in F_{j}} x_{t}+\left(-b_{i}+A\right)\right) \in \\
& \left.a_{i, l+2} p+\ldots+a_{i, k(i)} p\right\} .
\end{aligned}
$$

At $n=0$, hypotheses (I) and (II) hold directly, hypotheses (III), (V), and (VI) are vacuous, and hypothesis (IV) is the assertion that $-a_{0,0} x_{0}+\left(-b_{0}+A\right) \in a_{0,1} p+\ldots+a_{0, k(0)} p$.

For $i \in\{0,1, \ldots, n\}$ and $l \in\{0,1, \ldots, k(i)-1\}$, let

$$
\begin{aligned}
\mathcal{F}_{i, l}=\left\{\left(F_{0}, F_{1}, \ldots, F_{l}\right):\right. & F_{0}, F_{1}, \ldots, F_{l} \in \mathcal{P}_{f}(\{i, i+1, \ldots, n\}) \text { and } \\
& \left.F_{0}<F_{1}<\ldots<F_{l}\right\} .
\end{aligned}
$$

(Of course, if $l>n-i$, then $\mathcal{F}_{i, l}=\emptyset$.) For $i \in\{0,1, \ldots, n\}$, let

$$
E_{i}=\left\{\sum_{t \in F} x_{t}: \emptyset \neq F \subseteq\{0,1, \ldots, n\} \text { and } \min F=i\right\} .
$$

In the definition of $B_{n+1}$ below, we use the convention that $\bigcap \emptyset=\mathbb{N}$. So, for example, if $i \in\{0,1, \ldots, n\}, l \in\{0,1, \ldots, k(i)-2\}$, and $\mathcal{F}_{i, l}=\emptyset$, then one ignores the term

$$
\begin{gathered}
\left\{x \in \mathbb{N}:-a_{i, l+1} x+\left(-\sum_{j=0}^{l} a_{i, j} \sum_{t \in F_{j}} x_{t}+\left(-b_{i}+A\right)\right) \in\right. \\
\left.a_{i, l+2} p+\ldots+a_{i, k(i)} p\right\} .
\end{gathered}
$$

Let

$$
\begin{aligned}
B_{n+1}= & \left\{x \in \mathbb{N}:-a_{n+1,0} x+\left(-b_{n+1}+A\right) \in a_{n+1,1} p+\ldots+a_{n+1, k(n+1)} p\right\} \cap \\
& B_{n} \cap \bigcap_{i=0}^{n} \bigcap_{c \in E_{i}}\left(-c+B_{i}^{\star}\right) \cap \\
& \bigcap_{i=0}^{n} \bigcap_{\left(F_{0}, \ldots, F_{k(i)-1}\right) \in \mathcal{F}_{i, k(i)-1}}-\sum_{j=0}^{k(i)-1} a_{i, j} \sum_{t \in F_{j}} x_{t}+\left(-b_{i}+A\right) \cap \\
& \bigcap_{i=0}^{n} \bigcap_{l=0}^{k(i)-2} \bigcap_{\left(F_{0}, \ldots, F_{l}\right) \in \mathcal{F}_{i, l}} \\
& \left\{x \in \mathbb{N}:-a_{i, l+1} x+\left(-\sum_{j=0}^{l} a_{i, j} \sum_{t \in F_{j}} x_{t}+\left(-b_{i}+A\right)\right) \in\right. \\
& \left.a_{i, l+2} p+\ldots+a_{i, k(i)} p\right\} .
\end{aligned}
$$

To show that $B_{n+1} \in p$, we show that each set listed in its definition is in $p$. Since $-b_{n+1}+A \in a_{n+1,0} p+a_{n+1,1} p+\ldots+a_{n+1, k(n+1)} p$, we have that

$$
\left\{x \in \mathbb{N}:-a_{n+1,0} x+\left(-b_{n+1}+A\right) \in a_{n+1,1} p+\ldots+a_{n+1, k(n+1)} p\right\} \in p,
$$


and $B_{n} \in p$ by assumption. Given $i \in\{0,1, \ldots, n\}$ and $c \in E_{i}$, we have that $c \in B_{i}^{\star}$ by hypothesis (II) so $-c+B_{i}^{\star} \in p$.

Given $i \in\{0,1, \ldots, n\}$ and $\left(F_{0}, \ldots, F_{k(i)-1}\right) \in \mathcal{F}_{i, k(i)-1}$ we have by hypothesis (IV) that $-\sum_{j=0}^{k(i)-1} a_{i, j} \sum_{t \in F_{j}} x_{t}+\left(-b_{i}+A\right) \in a_{i, k(i)} p=p$.

Now let $i \in\{0,1, \ldots, n\}, l \in\{0,1, \ldots, k(i)-2\}$, and $\left(F_{0}, \ldots, F_{l}\right) \in \mathcal{F}_{i, l}$ be given. By hypothesis (IV),

$$
\begin{aligned}
-\sum_{j=0}^{l} a_{i, j} \sum_{t \in F_{j}} x_{t}+\left(-b_{i}+A\right) & \in a_{i, l+1} p+\ldots+a_{i, k(i)} p \\
& =a_{i, l+1} p+\left(a_{i, l+2} p+\ldots+a_{i, k(i)} p\right),
\end{aligned}
$$

so $\left\{x \in \mathbb{N}:-a_{i, l+1} x+\left(-\sum_{j=0}^{l} a_{i, j} \sum_{t \in F_{j}} x_{t}+\left(-b_{i}+A\right)\right) \in a_{i, l+2} p+\ldots+a_{i, k(i)} p\right\} \in p$.

Since $B_{n+1} \in p$, pick $x_{n+1} \in B_{n+1}^{\star}$.

Hypothesis (I) holds by assumption if $r \leqslant n$ and by construction if $r=n+1$. Similarly hypotheses (III), (V), and (VI) hold by assumption if $r<n$ and by construction if $r=n$.

To verify hypothesis (II), let $\emptyset \neq F \subseteq\{0,1, \ldots, n+1\}$ and let $i=\min F$. If $n+1 \notin F$, the statement holds by assumption and if $F=\{n+1\}$, this is the assertion that $x_{n+1} \in$ $B_{n+1}$. So assume that $\{n+1\} \subsetneq F$ and let $G=F \backslash\{n+1\}$. Then $\sum_{t \in G} x_{t} \in E_{i}$ so $x_{n+1} \in-\sum_{t \in G} x_{t}+B_{i}^{\star}$.

To verify hypothesis (IV), let $i \in\{0,1, \ldots, n\}$, let $l \in\{0,1, \ldots, k(i)-1\}$, and let $\left(F_{0}, \ldots, F_{l}\right) \in \mathcal{F}_{i, l}$. If $l=0$, let $i=\min F_{0}$; then by hypothesis (II), $\sum_{t \in F_{0}} x_{t} \in B_{i}$ so the conclusion holds by hypothesis (I). So assume that $l>0$, let $l^{\prime}=l-1$, and let $r=\max F_{l^{\prime}}$. Then $i \in\{0,1, \ldots, r\}, l^{\prime} \in\{0,1, \ldots, k(i)-2\}, F_{0}, F_{1}, \ldots, F_{l^{\prime}} \in \mathcal{P}_{f}(\{i, i+1 \ldots, r\})$ and $r<n+1$. Then by hypotheses (II) amd (III), $\sum_{t \in F_{l}} x_{t} \in B_{r+1}$ so the conclusion holds by hypothesis (VI).

The induction being complete, we have that $F S\left(\left\langle x_{n}\right\rangle_{n=0}^{\infty}\right) \subseteq A$ by hypotheses (II) and (III) and the fact that $B_{0} \subseteq A$. Finally, let $i \in \omega$ and let $F_{0}, F_{1}, \ldots, F_{k(i)}$ be given in $\mathcal{P}_{f}(\{i, i+1, \ldots\})$ such that $F_{0}<F_{1}<\ldots<F_{k(i)}$. Let $n=\min F_{k(i)}$ and $m=\max F_{k(i)-1}$. By hypotheses (II) and (III), $\sum_{t \in F_{k(i)}} x_{t} \in B_{n} \subseteq B_{m+1}$ so by hypothesis (V),

$$
\sum_{t \in F_{k(i)}} x_{t} \in-\sum_{j=0}^{k(i)-1} a_{i, j} \sum_{t \in F_{j}} x_{t}+\left(-b_{i}+A\right) .
$$

Thus, since $a_{i, k(i)}=1, b_{i}+\sum_{j=0}^{k(i)} a_{i, j} \sum_{t \in F_{j}} x_{t}+A$.

Corollary 6.4. Let $m \in \omega$ and for each $i \in\{0,1, \ldots, m\}$, let $k(i) \in \mathbb{N}$, let $\vec{a}_{i}=$ $\left\langle a_{i, 0}, a_{i, 1}, \ldots, a_{i, k(i)}\right\rangle$ be a compressed sequence in $\mathbb{Z} \backslash\{0\}$ with $a_{i, k(i)}=1$, and let $M_{i}$ be an $M T\left(\vec{a}_{i}\right)$-matrix. Let $\overline{0}$ and $\overline{1}$ be the length $\omega$ constant vectors. Then

$$
B=\left(\begin{array}{ccccc}
\overline{1} & \overline{0} & \ldots & \overline{0} & M_{0} \\
\overline{0} & \overline{1} & \ldots & \overline{0} & M_{1} \\
\vdots & \vdots & \ddots & \vdots & \vdots \\
\overline{0} & \overline{0} & \ldots & \overline{1} & M_{m} \\
\overline{0} & \overline{0} & \ldots & \overline{0} & \mathbf{F}
\end{array}\right)
$$

is centrally IPR. 
Proof. Let $A$ be a central set and pick a minimal idempotent $p$ such that $A \in p$. For $i>m$ let $\vec{a}_{i}=\langle 2,1\rangle$ (or any other reasonable choice) and let $\left\langle b_{n}\right\rangle_{n=0}^{\infty}$ and $\left\langle x_{n}\right\rangle_{n=0}^{\infty}$ be as guaranteed by Theorem 6.3 .

For $n<\omega$, let $y_{n}=x_{m+n}$. Then all entries of

$$
B\left(\begin{array}{c}
b_{0} \\
b_{1} \\
\vdots \\
b_{m} \\
\vec{y}
\end{array}\right)
$$

are in $A$.

What Theorem 6.3 is telling us is that, if we are allowed to add new variables (to represent the 'translation') then $\mathbf{F}$ is very far from being maximal. This motivates the following definition. We say that an IPR matrix $A$ is universally image maximal provided that whenever $B$ is an IPR matrix that image dominates $A$, then $A$ image dominates $B$

Is $\mathbf{D}$ universally image maximal? One might hope that the answer is yes, but it turns out that, similarly to Theorem 6.3 , one can actually extend $\mathbf{D}$ by a translate of what one might call a 'DHMT' system.

Definition 6.5. Let $k \in \mathbb{N}$, let $\vec{a}=\left\langle a_{0}, a_{1}, \ldots, a_{k}\right\rangle$ and for each $n<\omega$ let $Y_{n} \in \mathcal{P}_{f}(\mathbb{Q})$. Then $\operatorname{MT}\left(\vec{a},\left\langle Y_{n}\right\rangle_{n=0}^{\infty}\right)=\left\{\sum_{i=0}^{k} a_{i} \sum_{t \in F_{i}} x_{t}: F_{0}, F_{1}, \ldots, F_{k} \in \mathcal{P}_{f}(\omega), F_{0}<F_{1}<\ldots<\right.$ $F_{k}$ and $\left.x \in \mathrm{X}_{t \in \bigcup_{i=0}^{k} F_{i}} Y_{t}\right\}$.

Fix an enumeration $\left\langle B_{n}\right\rangle_{n=0}^{\infty}$ of the finite IPR matrices with rational entries. For each $n$, assume that $B_{n}$ is a $u(n) \times v(n)$ matrix.

Theorem 6.6. Let $p$ be a minimal idempotent in $\beta \mathbb{N}$ and let $A \in p$. There exist $b \in \mathbb{N}$ and a sequence $\left\langle Y_{n}\right\rangle_{n=0}^{\infty}$ in $\mathcal{P}_{f}(\mathbb{N})$ such that each $Y_{n}$ is the set of entries of an image of $B_{n}$ and $F S\left(\left\langle Y_{n}\right\rangle_{n=0}^{\infty}\right) \cup\left(b+M T\left(\langle 2,1\rangle,\left\langle Y_{n}\right\rangle_{n=0}^{\infty}\right)\right) \subseteq A$.

Proof. As in the proof of Lemma 6.1, pick $q \in \beta \mathbb{N}$ such that $p=q+2 p+p$ and pick $b \in \mathbb{N}$ such that $-b+A^{\star} \in 2 p+p$. Let $D=\left\{x \in A^{\star}:-2 x+\left(-b+A^{\star}\right) \in p\right\}$. Then $D \in p$.

Choose $\vec{x}(0) \in \mathbb{N}^{v(0)}$ such that, letting $Y_{0}$ be the set of entries of $B_{0} \vec{x}(0)$, we have $Y_{0} \subseteq D^{\star}$.

Inductively let $n \in \omega$ and assume that we have chosen $\vec{x}(k) \in \mathbb{N}^{v(k)}$ such that, letting $Y_{k}$ be the set of entries of $B_{k} \vec{x}(k)$, we have that

(1) $F S\left(\left\langle Y_{k}\right\rangle_{k=0}^{n}\right) \subseteq D^{\star}$ and

(2) if $n>0$, then $M T\left(\langle 2,1\rangle,\left\langle Y_{k}\right\rangle_{k=0}^{n}\right) \subseteq-b+A^{\star}$.

Now, if $x \in F S\left(\left\langle Y_{k}\right\rangle_{k=0}^{n}\right)$, then $-x+D^{\star} \in p$ and $-2 x+\left(-b+A^{\star}\right) \in p$. Also, if $n>0$ and $x \in M T\left(\langle 2,1\rangle,\left\langle Y_{k}\right\rangle_{k=0}^{n}\right)$, then $x \in\left(-b+A^{\star}\right)$ so $b+x \in A^{\star}$ and thus $-(b+x)+A^{\star} \in p$. 
Pick $\vec{x}(n+1) \in \mathbb{N}^{v(n+1)}$ such that

$$
\begin{aligned}
Y_{n+1} \subseteq & D^{\star} \cap \bigcap_{x \in F S\left(\left\langle Y_{k}\right\rangle_{k=0}^{n}\right)}\left(\left(-x+D^{\star}\right) \cap\left(-2 x+\left(-b+A^{\star}\right)\right)\right) \\
& \left.\cap \bigcap_{x \in M T\left(\langle 2,1\rangle,\left\langle Y_{k}\right\rangle_{k=0}^{n}\right)}\left(-(b+x)+A^{\star}\right)\right) .
\end{aligned}
$$

To see that $F S\left(\left\langle Y_{k}\right\rangle_{k=0}^{n+1}\right) \subseteq D^{\star}$, let $\emptyset \neq F \subseteq\{0,1, \ldots, n+1\}$ and let $x \in \times_{t \in F} Y_{t}$. If $n+1 \notin F$, we have that $\sum_{t \in F} x_{t} \in D^{\star}$ by hypothesis (1). If $F=\{n+1\}$, then $x_{n+1} \in Y_{n+1} \subseteq D^{\star}$. So assume that $\{n+1\} \subsetneq F$ and let $F^{\prime}=F \backslash\{n+1\}$. Then $x_{n+1} \in-\left(\sum_{t \in F^{\prime}} x_{t}\right)+D^{\star}$ so $\sum_{t \in F} x_{t} \in D^{\star}$.

To verify that $M T\left(\langle 2,1\rangle,\left\langle Y_{k}\right\rangle_{k=0}^{n+1}\right) \subseteq-b+A^{\star}$, let $F, H \in \mathcal{P}_{f}(\{1,2, \ldots, n+1\})$ such that $\max F<\min H$ and let $x \in \mathrm{X}_{k \in F \cup H} Y_{k}$. If $\max H<n+1$ the conclusion holds by the hypothesis (2), so assume that $n+1 \in H$. If $H=\{n+1\}$, then $x_{n+1} \in\left(-2 \sum_{t \in F} x_{t}+\right.$ $\left.\left(-b+A^{\star}\right)\right)$ so $\sum_{t \in F} 2 x_{t}+x_{n+1} \in-b+A^{\star}$.

Now assume that $\{n+1\} \subsetneq H$ and let $H^{\prime}=H \backslash\{n+1\}$. Then $x_{n+1} \in-\left(b+\sum_{t \in F} 2 x_{t}+\right.$ $\left.\sum_{t \in H^{\prime}} x_{t}\right)+A^{\star}$ so $\sum_{t \in F} 2 x_{t}+\sum_{t \in H} x_{t} \in-b+A^{\star}$ as required.

We remark that the analogue of Theorem 6.3 wherein $\left\langle x_{n}\right\rangle_{n=0}^{\infty}$ is replaced by $\left\langle Y_{n}\right\rangle_{n=0}^{\infty}$ remains valid with essentially the same proof.

We do not know any examples of universally image maximal systems.

Question 6.7. Does there exist a universally image maximal matrix?

\section{References}

[1] B. Barber, N. Hindman, and I. Leader. Partition regularity in the rationals. J. Comb. Theory (Series A), 120:1590-1599, 2013.

[2] B. Barber, N. Hindman, I. Leader, and D. Strauss. Partition regularity without the columns property. Proc. Amer. Math. Soc., to appear. doi : 10.1090/S0002-9939-2015-12519-1

[3] W. Deuber. Partitionen und lineare Gleichungssysteme. Math. Z. 133:109-123, 1973.

[4] W. Deuber and N. Hindman. Partitions and sums of $(m, p, c)$-sets. J. Comb. Theory (Series A), 45:300-302, 1987.

[5] W. Deuber, N. Hindman, I. Leader, and H. Lefmann. Infinite partition regular matrices. Combinatorica, 15:333-355, 1995.

[6] N. Hindman. Finite sums from sequences within cells of a partition of $\mathbb{N}$. J. Comb. Theory (Series A), 17:1-11, 1974.

[7] N. Hindman and I. Leader. Image partition regularity of matrices. Comb. Prob. and Comp. 2:437-463, 1993.

[8] N. Hindman, I. Leader, and D. Strauss. Infinite partition regular matrices - solutions in central sets. Trans. Amer. Math. Soc., 355:1213-1235, 2003.

[9] N. Hindman, I. Leader, and D. Strauss. Separating Milliken-Taylor systems with negative entries. Proc. Edinburgh Math. Soc., 46:45-61, 2003. 
[10] N. Hindman and D. Strauss. Algebra in the Stone-Čech compactification: theory and applications, 2nd edition. Walter de Gruyter \& Co., Berlin, 2012.

[11] K. Milliken. Ramsey's Theorem with sums or unions. J. Comb. Theory (Series A), 18:276-290, 1975.

[12] R. Rado. Studien zur Kombinatorik. Math. Z., 36:424-470, 1933.

[13] I. Schur. Über die Kongruenz $x^{m}+y^{m}=z^{m}(\bmod p)$. Jahresbericht der Deutschen Math.-Verein., 25:114-117, 1916.

[14] A. Taylor. A canonical partition relation for finite subsets of $\omega$. J. Comb. Theory (Series A), 21:137-146, 1976.

[15] B. van der Waerden. Beweis einer Baudetschen Vermutung. Nieuw Arch. Wiskunde, 19:212-216, 1927. 